\title{
VARIABLE SELECTION WITH HAMMING LOSS
}

\author{
By CRistina ButuCEA ${ }^{* \dagger, 1}$, MOHAMEd NdaOUd ${ }^{\dagger, 1}$, \\ NATAlia A. Stepanova ${ }^{\ddagger, 2}$ AND AlexandRe B. TsybaKOV ${ }^{\dagger, 1,3}$ \\ Université Paris-Est Marne-la-Vallée*, CREST, ENSAE ${ }^{\dagger}$ and \\ Carleton University:
}

\begin{abstract}
We derive nonasymptotic bounds for the minimax risk of variable selection under expected Hamming loss in the Gaussian mean model in $\mathbb{R}^{d}$ for classes of at most $s$-sparse vectors separated from 0 by a constant $a>0$. In some cases, we get exact expressions for the nonasymptotic minimax risk as a function of $d, s, a$ and find explicitly the minimax selectors. These results are extended to dependent or non-Gaussian observations and to the problem of crowdsourcing. Analogous conclusions are obtained for the probability of wrong recovery of the sparsity pattern. As corollaries, we derive necessary and sufficient conditions for such asymptotic properties as almost full recovery and exact recovery. Moreover, we propose data-driven selectors that provide almost full and exact recovery adaptively to the parameters of the classes.
\end{abstract}

1. Introduction. In recent years, the problem of variable selection in highdimensional regression models has been extensively studied from the theoretical and computational viewpoints. In making effective high-dimensional inference, sparsity plays a key role. With regard to variable selection in sparse highdimensional regression, the Lasso, Dantzig selector, other penalized techniques as well as marginal regression were analyzed in detail; see, for example, [13, 16, 20 $22,24,25,27,28]$ and the references cited therein. Several other recent papers deal with sparse variable selection in nonparametric regression; see, for example, $[5,9,11,15,18]$.

In this paper, we study the problem of variable selection in the Gaussian sequence model

$$
X_{j}=\theta_{j}+\sigma \xi_{j}, \quad j=1, \ldots, d,
$$

Received December 2015; revised March 2017.

${ }^{1}$ Supported by GENES and by the French National Research Agency (ANR) under the grants IPANEMA (ANR-13-BSH1-0004-02), and Labex ECODEC (ANR-11-LABEX-0047).

${ }^{2}$ Supported by an NSERC grant.

${ }^{3}$ Supported by the "Chaire Economie et Gestion des Nouvelles Données", under the auspices of Institut Louis Bachelier, Havas-Media and Paris-Dauphine.

MSC2010 subject classifications. 62G05, 62G08, 62G20.

Key words and phrases. Adaptive variable selection, almost full recovery, exact recovery, Hamming loss, minimax selectors, nonasymptotic minimax selection bounds, phase transitions. 
where $\xi_{1}, \ldots, \xi_{d}$ are i.i.d. standard Gaussian random variables, $\sigma>0$ is the noise level, and $\theta=\left(\theta_{1}, \ldots, \theta_{d}\right)$ is an unknown vector of parameters to be estimated. We assume that $\theta$ is $(s, a)$-sparse, which is understood in the sense that $\theta$ belongs to one of the following sets:

$\Theta_{d}(s, a)=\left\{\theta \in \mathbb{R}^{d}\right.$ : there exists a set $S \subseteq\{1, \ldots, d\}$ with at most $s$ elements such that $\left|\theta_{j}\right| \geq a$ for all $j \in S$, and $\theta_{j}=0$ for all $\left.j \notin S\right\}$

or

$\Theta_{d}^{+}(s, a)=\left\{\theta \in \mathbb{R}^{d}:\right.$ there exists a set $S \subseteq\{1, \ldots, d\}$ with at most $s$ elements such that $\theta_{j} \geq a$ for all $j \in S$, and $\theta_{j}=0$ for all $\left.j \notin S\right\}$.

Here, $a>0$ and $s \in\{1, \ldots, d\}$ are given constants.

We study the problem of selecting the relevant components of $\theta$, that is, of estimating the vector

$$
\eta=\eta(\theta)=\left(I\left(\theta_{j} \neq 0\right)\right)_{j=1, \ldots, d},
$$

where $I(\cdot)$ is the indicator function. As estimators of $\eta$, we consider any measurable functions $\hat{\eta}=\hat{\eta}\left(X_{1}, \ldots, X_{n}\right)$ of $\left(X_{1}, \ldots, X_{n}\right)$ taking values in $\{0,1\}^{d}$. Such estimators will be called selectors. We characterize the loss of a selector $\hat{\eta}$ as an estimator of $\eta$ by the Hamming distance between $\hat{\eta}$ and $\eta$, that is, by the number of positions at which $\hat{\eta}$ and $\eta$ differ:

$$
|\hat{\eta}-\eta| \triangleq \sum_{j=1}^{d}\left|\hat{\eta}_{j}-\eta_{j}\right|=\sum_{j=1}^{d} I\left(\hat{\eta}_{j} \neq \eta_{j}\right) .
$$

Here, $\hat{\eta}_{j}$ and $\eta_{j}=\eta_{j}(\theta)$ are the $j$ th components of $\hat{\eta}$ and $\eta=\eta(\theta)$, respectively. The expected Hamming loss of a selector $\hat{\eta}$ is defined as $\mathbf{E}_{\theta}|\hat{\eta}-\eta|$, where $\mathbf{E}_{\theta}$ denotes the expectation with respect to the distribution $\mathbf{P}_{\theta}$ of $\left(X_{1}, \ldots, X_{n}\right)$ satisfying (1). Another well-known risk measure is the probability of wrong recovery $\mathbf{P}_{\theta}(\hat{S} \neq S(\theta))$, where $\hat{S}=\left\{j: \hat{\eta}_{j}=1\right\}$ and $S(\theta)=\left\{j: \eta_{j}(\theta)=1\right\}$. It can be viewed as the Hamming distance with an indicator loss and is related to the expected Hamming loss as follows:

$$
\mathbf{P}_{\theta}(\hat{S} \neq S(\theta))=\mathbf{P}_{\theta}(|\hat{\eta}-\eta| \geq 1) \leq \mathbf{E}_{\theta}|\hat{\eta}-\eta| .
$$

In view of the last inequality, bounding the expected Hamming loss provides a stronger result than bounding the probability of wrong recovery.

Most of the literature on variable selection in high dimensions focuses on the recovery of the sparsity pattern, that is, on constructing selectors such that the probability $\mathbf{P}_{\theta}(\hat{S} \neq S(\theta))$ is close to 0 in some asymptotic sense (see, e.g., [20-22, $24,25,27,28])$. These papers consider high-dimensional linear regression settings with deterministic or random covariates. In particular, for the sequence model (1), 
one gets that if $a>C \sigma \sqrt{\log d}$ for some $C>0$ large enough, then there exist selectors such that $\mathbf{P}_{\theta}(\hat{S} \neq S(\theta))$ tends to 0 , while this is not the case if $a<c \sigma \sqrt{\log d}$ for some $c>0$ small enough. More insight into variable selection was provided in $[13,16]$ by considering a Hamming risk close to the one we have defined above. Assuming that $s \sim d^{1-\beta}$ for some $\beta \in(0,1)$, the papers [13, 16] establish an asymptotic in $d$ "phase diagram" that partitions the parameter space into three regions called the exact recovery, almost full recovery, and no recovery regions. This is done in a Bayesian setup for the linear regression model with i.i.d. Gaussian covariates and random $\theta$. Note also that in $[13,16]$ the knowledge of $\beta$ is required to construct the selectors, so that in this sense the methods are not adaptive. The selectors are of the form $\hat{\eta}_{j}=I\left(\left|X_{j}\right| \geq t\right)$ with threshold $t=\tau(\beta) \sigma \sqrt{\log d}$ for some function $\tau(\cdot)>0$. More recently, these asymptotic results were extended to a combined minimax-Bayes Hamming risk on a certain class of vectors $\theta$ in [17].

The present paper makes further steps in the analysis of variable selection with a Hamming loss initiated in $[13,16]$. Unlike $[13,16]$, we study the sequence model (1) rather than Gaussian regression and analyze the behavior of the minimax risk rather than that of the Bayes risk with a specific prior. Furthermore, we consider not only $s \sim d^{1-\beta}$ but general $s$ and derive nonasymptotic results that are valid for any sample size. Remarkably, we get an exact expression for the nonasymptotic minimax risk of separable (coordinate-wise) selectors and find explicitly the separable minimax selectors. Finally, we construct data-driven selectors that are simultaneously adaptive to the parameters $a$ and $s$.

Specifically, we consider the minimax risk

$$
\inf _{\tilde{\eta}} \sup _{\theta \in \Theta} \frac{1}{S} \mathbf{E}_{\theta}|\tilde{\eta}-\eta|
$$

for $\Theta=\Theta_{d}(s, a)$ and $\Theta=\Theta_{d}^{+}(s, a)$, where $\inf _{\tilde{\eta}}$ denotes the infimum over all selectors $\tilde{\eta}$. In Section 2, for both classes $\Theta=\Theta_{d}(s, a)$ and $\Theta=\Theta_{d}^{+}(s, a)$ we find the upper and lower bounds of the minimax risks and derive minimax selectors for any fixed $d, s, a>0$ such that $s<d$. For $\Theta=\Theta_{d}(s, a)$, we also propose another selector attaining the lower bound risk up to the factor 2. Interestingly, the thresholds that correspond to the minimax optimal selectors do not have the classical form $A \sigma \sqrt{\log d}$ for some $A>0$; the optimal threshold is a function of $a$ and $s$. Analogous minimax results are obtained for the risk measured by the probability of wrong recovery $\mathbf{P}_{\theta}(\hat{S} \neq S(\theta))$. Section 3 considers extensions of the nonasymptotic minimax theorems of Section 2 to settings with non-Gaussian or dependent observations. In Section 4, as asymptotic corollaries of these results, we establish sharp conditions under which exact and almost full recovery are achievable. Section 5 is devoted to the construction of adaptive selectors that achieve almost full and exact recovery without the knowledge of the parameters $a$ and $s$. Most of the proofs are given in the Appendix. 
Finally, note that quite recently several papers have studied the expected Hamming loss in other problems of variable selection. Asymptotic behavior of the minimax risk analogous to (3) for classes $\Theta$ different from the sparsity classes that we consider here was analyzed in [9] and without the normalizing factor $1 / s$ in [15]. Oracle inequalities for Hamming risks in the problem of multiple classification under sparsity constraints are established in [23]. The paper [26] introduces an asymptotically minimax approach based on the Hamming loss in the problem of community detection in networks.

2. Nonasymptotic minimax selectors. In what follows, we assume that $s<d$. We first consider minimax variable selection for the class $\Theta_{d}^{+}(s, a)$. For this class, we will use a selector $\hat{\eta}^{+}$with the components

$$
\hat{\eta}_{j}^{+}=I\left(X_{j} \geq t\right), \quad j=1, \ldots, d,
$$

where the threshold is defined by

$$
t=\frac{a}{2}+\frac{\sigma^{2}}{a} \log \left(\frac{d}{s}-1\right) .
$$

Set

$\Psi_{+}(d, s, a)=\left(\frac{d}{s}-1\right) \Phi\left(-\frac{a}{2 \sigma}-\frac{\sigma}{a} \log \left(\frac{d}{s}-1\right)\right)+\Phi\left(-\frac{a}{2 \sigma}+\frac{\sigma}{a} \log \left(\frac{d}{s}-1\right)\right)$,

where $\Phi(\cdot)$ denotes the standard Gaussian cumulative distribution function.

THEOREM 2.1. For any $a>0$ and $s \leq d / 2$, the selector $\hat{\eta}^{+}$in (4) with the threshold t defined in (5) satisfies

$$
\sup _{\theta \in \Theta_{d}^{+}(s, a)} \frac{1}{s} \mathbf{E}_{\theta}\left|\hat{\eta}^{+}-\eta\right| \leq \Psi_{+}(d, s, a) .
$$

The proof is given in the Appendix.

A selector $\tilde{\eta}=\left(\tilde{\eta}_{1}, \ldots, \tilde{\eta}_{d}\right)$ will be called separable if its $j$ th component $\tilde{\eta}_{j}$ depends only on $X_{j}$ for all $j=1, \ldots, d$. We denote by $\mathcal{T}$ the set of all separable selectors.

The next theorem gives a lower bound on the minimax risk showing that the upper bound in Theorem 2.1 is tight over separable selectors.

THEOREM 2.2. For any $a>0$ and $s<d$, we have

$$
\inf _{\tilde{\eta} \in \mathcal{T}} \sup _{\theta \in \Theta_{d}^{+}(s, a)} \frac{1}{s} \mathbf{E}_{\theta}|\tilde{\eta}-\eta| \geq \Psi_{+}(d, s, a)
$$


where $\inf _{\tilde{\eta} \in \mathcal{T}}$ denotes the infimum over all separable selectors $\tilde{\eta}$. Moreover, for any $s^{\prime}$ in $(0, s]$, we have

$$
\inf _{\tilde{\eta}} \sup _{\theta \in \Theta_{d}^{+}(s, a)} \frac{1}{s} \mathbf{E}_{\theta}|\tilde{\eta}-\eta| \geq \frac{s^{\prime}}{s} \Psi_{+}(d, s, a)-\frac{4 s^{\prime}}{s} \exp \left(-\frac{\left(s-s^{\prime}\right)^{2}}{2 s}\right),
$$

where $\inf _{\tilde{\eta}}$ denotes the infimum over all selectors $\tilde{\eta}$.

The proof of the first inequality of Theorem 2.2 is given in the Appendix, while the proof of the second inequality is given in the Supplementary material [8].

As a straightforward corollary of Theorems 2.1 and 2.2, we obtain that the estimator $\hat{\eta}^{+}$is minimax among the separable selectors in the exact sense for the class $\Theta_{d}^{+}(s, a)$ and the minimax risk satisfies

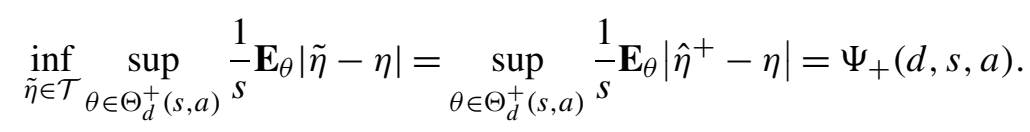

Remarkably, this holds under no assumptions on $d, s, a$ except for, of course, some minimal conditions under which the problem ever makes sense: $a>0$ and $s \leq d / 2$. Analogous non-asymptotic minimax result is valid for the class

$$
\begin{aligned}
\Theta_{d}^{-}(s, a)= & \left\{\theta \in \mathbb{R}^{d}: \text { there exists a set } S \subseteq\{1, \ldots, d\} \text { with at most } s\right. \text { elements } \\
& \text { such that } \left.\theta_{j} \leq-a \text { for all } j \in S, \text { and } \theta_{j}=0 \text { for all } j \notin S\right\} .
\end{aligned}
$$

We omit details here.

Next, consider the class $\Theta_{d}(s, a)$. A direct analog of $\hat{\eta}^{+}$for $\Theta_{d}(s, a)$ is a selector $\hat{\eta}$ with the components

$$
\hat{\eta}_{j}=I\left(\left|X_{j}\right| \geq t\right), \quad j=1, \ldots, d,
$$

where the threshold $t$ is defined in (5). Set

$$
\begin{aligned}
\Psi(d, s, a)= & \left(\frac{d}{s}-1\right) \Phi\left(-\frac{a}{2 \sigma}-\frac{\sigma}{a} \log \left(\frac{d}{s}-1\right)\right) \\
& +\Phi\left(-\left(\frac{a}{2 \sigma}-\frac{\sigma}{a} \log \left(\frac{d}{s}-1\right)\right)_{+}\right),
\end{aligned}
$$

where $x_{+}=\max (x, 0)$. Note that

$$
\Psi(d, s, a) \leq \Psi_{+}(d, s, a) .
$$

We have the following bound.

THEOREM 2.3. For any $a>0$ and $s \leq d / 2$, the selector $\hat{\eta}$ in (8) with the threshold $t$ defined in (5) satisfies

$$
\sup _{\theta \in \Theta_{d}(s, a)} \frac{1}{s} \mathbf{E}_{\theta}|\hat{\eta}-\eta| \leq 2 \Psi(d, s, a) .
$$


The proof is given in the Appendix.

For the minimax risk on the class $\Theta_{d}(s, a)$, we have the following corollary, which is an immediate consequence of Theorems 2.2, 2.3 and inequality (9).

COROLlary 2.1. For any $a>0$ and $s \leq d / 2$, the selector $\hat{\eta}$ in (8) with the threshold $t$ defined in (5) satisfies

$$
\sup _{\theta \in \Theta_{d}(s, a)} \mathbf{E}_{\theta}|\hat{\eta}-\eta| \leq 2 \inf _{\tilde{\eta} \in \mathcal{T}} \sup _{\theta \in \Theta_{d}(s, a)} \mathbf{E}_{\theta}|\tilde{\eta}-\eta| .
$$

Thus, the risk of the thresholding estimator (8) cannot be greater than the minimax risk of separable selectors over the class $\Theta_{d}(s, a)$ multiplied by 2 .

We turn now to exact minimax variable selection over the class $\Theta_{d}(s, a)$. Consider a selector $\bar{\eta}=\left(\bar{\eta}_{1}, \ldots, \bar{\eta}_{d}\right)$ with the components

$$
\bar{\eta}_{j}=I\left(\log \left(\cosh \left(\frac{a X_{j}}{\sigma^{2}}\right)\right) \geq t\right), \quad j=1, \ldots, d,
$$

where the threshold is defined by

$$
t=\frac{a^{2}}{2 \sigma^{2}}+\log \left(\frac{d}{s}-1\right) .
$$

Set

$$
\begin{aligned}
\bar{\Psi}(d, s, a)= & \left(\frac{d}{s}-1\right) \mathbf{P}\left(e^{-\frac{a^{2}}{2 \sigma^{2}}} \cosh \left(\frac{a \xi}{\sigma}\right) \geq \frac{d}{s}-1\right) \\
& +\mathbf{P}\left(e^{-\frac{a^{2}}{2 \sigma^{2}}} \cosh \left(\frac{a \xi}{\sigma}+\frac{a^{2}}{\sigma^{2}}\right)<\frac{d}{s}-1\right),
\end{aligned}
$$

where $\xi$ denotes a standard Gaussian random variable. Our aim is to show that $\bar{\Psi}(d, s, a)$ is the minimax risk of variable selection under the Hamming loss over the class $\Theta_{d}(s, a)$ and that it is nearly achieved by the selector in (12). We first prove that $\bar{\Psi}(d, s, a) d /(d-s)$ is an upper bound on the maximum risk of the selector (12).

THEOREM 2.4. For any $a>0$ and $s<d$, the selector $\bar{\eta}$ in (12) with the threshold t defined in (13) satisfies

$$
\sup _{\theta \in \Theta_{d}(s, a)} \frac{1}{s} \mathbf{E}_{\theta}|\bar{\eta}-\eta| \leq \bar{\Psi}(d, s, a) \frac{d}{d-s} .
$$

The next theorem establishes the lower bound over separable selectors on the minimax risk associated to the upper bound in Theorem 2.4. 
THEOREM 2.5. For any $a>0$ and $s<d$, we have

$$
\inf _{\tilde{\eta} \in \mathcal{T}} \sup _{\theta \in \Theta_{d}(s, a)} \frac{1}{s} \mathbf{E}_{\theta}|\tilde{\eta}-\eta| \geq \bar{\Psi}(d, s, a),
$$

where $\inf _{\tilde{\eta} \in \mathcal{T}}$ denotes the infimum over all separable selectors $\tilde{\eta}$.

Finally, we show how the above nonasymptotic minimax results can be extended to the probability of wrong recovery. For any selector $\tilde{\eta}$, we denote by $S_{\tilde{\eta}}$ the selected set of indices: $S_{\tilde{\eta}}=\left\{j: \tilde{\eta}_{j}=1\right\}$.

THEOREM 2.6. For any $a>0$ and $s \leq d / 2$, the selectors $\hat{\eta}$ in (8) and $\hat{\eta}^{+}$in (4) with the threshold $t$ defined in (5), and the selector $\bar{\eta}$ in (12) with the threshold t defined in (13) satisfy

$$
\begin{aligned}
& \sup _{\theta \in \Theta_{d}^{+}(s, a)} \mathbf{P}_{\theta}\left(S_{\hat{\eta}^{+}} \neq S(\theta)\right) \leq s \Psi_{+}(d, s, a), \\
& \sup _{\theta \in \Theta_{d}(s, a)} \mathbf{P}_{\theta}\left(S_{\bar{\eta}} \neq S(\theta)\right) \leq s \bar{\Psi}(d, s, a) \frac{d}{d-s}
\end{aligned}
$$

and

$$
\sup _{\theta \in \Theta_{d}(s, a)} \mathbf{P}_{\theta}\left(S_{\hat{\eta}} \neq S(\theta)\right) \leq 2 s \Psi(d, s, a) .
$$

Furthermore,

$$
\inf _{\tilde{\eta} \in \mathcal{T}} \sup _{\theta \in \Theta_{d}^{+}(s, a)} \mathbf{P}_{\theta}\left(S_{\tilde{\eta}} \neq S(\theta)\right) \geq \frac{s \Psi_{+}(d, s, a)}{1+s \Psi_{+}(d, s, a)}
$$

and

$$
\inf _{\tilde{\eta} \in \mathcal{T}} \sup _{\theta \in \Theta_{d}(s, a)} \mathbf{P}_{\theta}\left(S_{\tilde{\eta}} \neq S(\theta)\right) \geq \frac{s \bar{\Psi}(d, s, a)}{1+s \bar{\Psi}(d, s, a)} .
$$

The proof is given in the Appendix.

Although Theorem 2.6 does not provide the exact minimax solution, it implies sharp minimaxity in asymptotic sense. Indeed, an interesting case is when the minimax risk in Theorem 2.6 goes to 0 as $d \rightarrow \infty$. Assuming that $s$ and $a$ are functions of $d$, this corresponds to $s \Psi_{+}(d, s, a) \rightarrow 0$ as $d \rightarrow \infty$. In this natural asymptotic setup, the upper and lower bounds of Theorem 2.6 for the class $\Theta_{d}^{+}(s, a)$ are sharp. The same for the class $\Theta_{d}(s, a)$, if $s$ and $a$ are such that $s \bar{\Psi}(d, s, a) \rightarrow 0$ and that $s / d \rightarrow 0$. We discuss this issue in Section 4; cf. Theorem 4.5.

Remark 2.1. Papers $[13,16,17]$ use a different Hamming loss defined in terms of vectors of signs. In our setting, this would mean considering not $|\hat{\eta}-\eta|$ 
but the following loss: $\sum_{j=1}^{d} I\left(\operatorname{sign}\left(\hat{\theta}_{j}\right) \neq \operatorname{sign}\left(\theta_{j}\right)\right)$, where $\hat{\theta}_{j}$ is an estimator of $\theta_{j}$ and $\operatorname{sign}(x)=I(x>0)-I(x<0)$. Theorems of this section are easily adapted to such a loss, but in this case the corresponding expressions for the nonasymptotic risk contain additional terms and we do not obtain exact minimax solutions as above. On the other hand, these additional terms are smaller than $\Psi(d, s, a)$ and $\Psi_{+}(d, s, a)$, and in the asymptotic analysis, such as the one performed in Sections 4 and 5 , can often be neglected. Thus, in many cases, one gets the same asymptotic results for both losses. We do not discuss this issue in more detail here.

3. Generalizations and extensions. Before proceeding to asymptotic corollaries, we discuss some generalizations and extensions of the nonasymptotic results of Section 2.

3.1. Dependent observations. It is easy to see that Theorems 2.1 and 2.3 do not use any information on the dependence between the observations, and thus remain valid for dependent $X_{j}$. Furthermore, a minimax optimality property within the class of separable selectors holds under dependence as well. To be specific, denote by $\mathcal{N}_{d}(\theta, \Sigma)$ the $d$-dimensional Gaussian distribution with mean $\theta$ and covariance matrix $\Sigma$. Assume that the distribution $\mathbf{P}$ of $\left(X_{1}, \ldots, X_{d}\right)$ belongs to the class

$$
\mathcal{P}_{d}^{+}\left(s, a, \sigma^{2}\right)=\left\{\mathcal{N}_{d}(\theta, \Sigma): \theta \in \Theta_{d}^{+}(s, a), \sigma_{i i}=\sigma^{2}, \text { for all } i=1, \ldots, d\right\},
$$

where we denote by $\sigma_{i i}$ the diagonal entries of $\Sigma$. Note that, for distributions in this class, $\Sigma$ can be any covariance matrix with constant diagonal elements.

THEOREM 3.1. For any $a>0$ and $s \leq d / 2$, and for the selector $\hat{\eta}^{+}$in (4) with the threshold $t$ defined in (5) we have

$$
\inf _{\tilde{\eta} \in \mathcal{T}} \sup _{\mathbf{P} \in \mathcal{P}_{d}^{+}\left(s, a, \sigma^{2}\right)} \mathbf{E}_{\mathbf{P}}|\tilde{\eta}-\eta|=\sup _{\mathbf{P} \in \mathcal{P}_{d}^{+}\left(s, a, \sigma^{2}\right)} \mathbf{E}_{\mathbf{P}}\left|\hat{\eta}^{+}-\eta\right|=s \Psi_{+}(d, s, a),
$$

where $\inf _{\tilde{\eta} \in \mathcal{T}}$ denotes the infimum over all separable selectors $\tilde{\eta}$, and $\mathbf{E}_{\mathbf{P}}$ denotes the expectation with respect to $\mathbf{P}$. Moreover, for any $s^{\prime}$ in $(0, s]$, we have

$$
\inf _{\tilde{\eta}} \sup _{\mathbf{P} \in \mathcal{P}_{d}^{+}\left(s, a, \sigma^{2}\right)} \mathbf{E}_{\mathbf{P}}|\tilde{\eta}-\eta| \geq s^{\prime} \Psi_{+}(d, s, a)-4 s^{\prime} \exp \left(-\frac{\left(s-s^{\prime}\right)^{2}}{2 s}\right),
$$

where $\inf _{\tilde{\eta}}$ denotes the infimum over all selectors $\tilde{\eta}$.

Proof. The upper bound $\Psi_{+}(d, s, a)$ on the minimax risk follows from the fact that the proofs of Theorems 2.1 and 2.3 are not affected by the dependence. Indeed, both the selector and the Hamming loss proceed coordinate-wisely. The lower bound on the minimax risk follows from Theorem 2.2 after observing that the maximum over $\mathcal{P}_{d}^{+}\left(s, a, \sigma^{2}\right)$ is greater than the maximum over the subfamily 
of Gaussian vectors with independent entries $\left\{\mathcal{N}_{d}\left(\theta, \sigma^{2} I_{d}\right): \theta \in \Theta_{d}^{+}(s, a)\right\}$, where $I_{d}$ is the $d \times d$ identity matrix.

An interesting consequence of Theorem 3.1 and of (7) is that the model with independent $X_{j}$ is the least favorable model, in the exact nonasymptotic sense, for the problem of variable selection with Hamming loss on the class of vectors $\Theta_{d}^{+}(s, a)$.

This fact was also noticed and discussed in [14] for the detection problem. That paper considers the Gaussian model with covariance matrix $\Sigma$ that is not necessarily a diagonal matrix. It is shown that faster detection rates are achieved in the case of dependent observations (under some assumptions) than in the case of independent data. It would be interesting to extend these results to the variable selection problem in hand.

3.2. Non-Gaussian models. As a building block for extension to non-Gaussian observations, we first consider the following simple model. We observe independent random variables $X_{1}, \ldots, X_{d}$ with values in a measurable space $(\mathcal{X}, \mathcal{U})$ such that at most $s$ among them are distributed according to the probability distribution $P_{1}$ and the other are distributed according to the probability distribution $P_{0}$. We assume that $P_{0} \neq P_{1}$. Let $f_{0}$ and $f_{1}$ be densities of $P_{0}$ and $P_{1}$ with respect to some dominating measure. Denote by $\eta=\left(\eta_{1}, \ldots, \eta_{d}\right)$ the vector such that $\eta_{j}=1$ if the distribution of $X_{j}$ is $P_{1}$ and $\eta_{j}=0$ if it is $P_{0}$. Define $\Theta_{d}(s)$ as the set of all vectors $\eta \in\{0,1\}^{d}$ with at most $s$ non-zero components. For any fixed $\eta$, we denote by $\mathbf{E}_{\eta}$ the expectation with respect to the distribution of $\left(X_{1}, \ldots, X_{d}\right)$. Consider the selector $\hat{\eta}=\left(\hat{\eta}_{1}, \ldots, \hat{\eta}_{d}\right)$, where

$$
\hat{\eta}_{j}=I\left(s f_{1}\left(X_{j}\right) \geq(d-s) f_{0}\left(X_{j}\right)\right), \quad j=1, \ldots, d .
$$

THEOREM 3.2. For any $s<d$, the selector $\hat{\eta}$ in (19) satisfies

$$
\sup _{\eta \in \Theta_{d}(s)} \mathbf{E}_{\eta} \frac{1}{s}|\hat{\eta}-\eta| \leq \Psi(d, s) \frac{d}{d-s}
$$

and, for any $s^{\prime}$ in $(0, s]$,

$$
\inf _{\tilde{\eta}} \sup _{\eta \in \Theta_{d}(s)} \frac{1}{s} \mathbf{E}_{\eta}|\tilde{\eta}-\eta| \geq \frac{s^{\prime}}{s} \Psi(d, s)-\frac{4 s^{\prime}}{s} \exp \left(-\frac{\left(s-s^{\prime}\right)^{2}}{2 s}\right),
$$

where $\inf _{\tilde{\eta}}$ denotes the infimum over all selectors, and

$$
\begin{aligned}
\Psi=\Psi(d, s)= & P_{1}\left(s f_{1}\left(X_{1}\right)<(d-s) f_{0}\left(X_{1}\right)\right) \\
& +\left(\frac{d}{s}-1\right) P_{0}\left(s f_{1}\left(X_{1}\right) \geq(d-s) f_{0}\left(X_{1}\right)\right) .
\end{aligned}
$$


The proof is given in the Supplementary material [8].

Suppose now that instead of two measures $P_{0}$ and $P_{1}$ we have a parametric family of probability measures $\left\{\mathbb{P}_{a}, a \in \mathcal{U}\right\}$ where $\mathcal{U} \subseteq \mathbb{R}$. Let $\mathrm{f}_{a}$ be a density of $\mathbb{P}_{a}$ with respect to some dominating measure. Recall that the family $\left\{\mathrm{f}_{a}, a \in \mathcal{U}\right\}$ is said to have the Monotone Likelihood Ratio (MLR) property if, for all $a_{0}, a_{1}$ in $\mathcal{U}$ such that $a_{0}<a_{1}$, the $\log$-likelihood ratio $\log \left(\mathfrak{f}_{a_{1}}(X) / \mathfrak{f}_{a_{0}}(X)\right)$ is an increasing function of $X$. In particular, this implies (cf. [19], Lemma 3.4.2) that $\left\{\mathrm{f}_{a}, a \in \mathcal{U}\right\}$ is a stochastically ordered family, that is,

$$
F_{a}(x) \geq F_{a^{\prime}}(x) \quad \text { for all } x \text { if } a<a^{\prime},
$$

where $F_{a}$ is the cumulative distribution function corresponding to $\mathrm{f}_{a}$. Using these facts, we generalize the nonasymptotic results of the previous section in two ways. First, we allow for not necessarily Gaussian distributions and second, instead of the set of parameters $\Theta_{d}^{+}(s, a)$, we consider the following set with two restrictions:

$$
\begin{aligned}
\Theta_{d}^{+}\left(s, a_{0}, a_{1}\right)= & \left\{\theta \in \mathbb{R}^{d}: \exists \text { a set } S \subseteq\{1, \ldots, d\} \text { with at most } s\right. \text { elements } \\
& \text { such that } \left.\theta_{j} \geq a_{1} \text { for all } j \in S, \text { and } \theta_{j} \leq a_{0} \text { for all } j \notin S\right\},
\end{aligned}
$$

where $a_{0}<a_{1}$. We assume that $X_{j}$ is distributed with density $\mathrm{f}_{\theta_{j}}$ for $j=1, \ldots, d$, and $X_{1}, \ldots, X_{d}$ are independent. In the next theorem, $\mathbf{E}_{\theta}$ is the expectation with respect to the distribution of such $X_{1}, \ldots, X_{d}$. In what follows, we use the notation $f_{j}=\mathrm{f}_{a_{j}}, j=0,1$.

THEOREM 3.3. Let $\left\{\mathrm{f}_{a}, a \in \mathcal{U}\right\}$ be a family with the MLR property, and let $a_{0}, a_{1} \in \mathcal{U}$ be such that $a_{0}<a_{1}$. Set $f_{0}=\mathrm{f}_{a_{0}}$ and $f_{1}=\mathrm{f}_{a_{1}}$, then, for any $s<d$, the selector $\hat{\eta}$ in (19) satisfies

$$
\sup _{\theta \in \Theta_{d}^{+}\left(s, a_{0}, a_{1}\right)} \frac{1}{s} \mathbf{E}_{\theta}|\hat{\eta}-\eta| \leq \Psi(d, s) \frac{d}{d-s},
$$

and, for any $s^{\prime}$ in $(0, s]$,

$$
\inf _{\tilde{\eta}} \sup _{\theta \in \Theta_{d}^{+}\left(s, a_{0}, a_{1}\right)} \frac{1}{s} \mathbf{E}_{\theta}|\tilde{\eta}-\eta| \geq \frac{s^{\prime}}{s} \Psi(d, s)-\frac{4 s^{\prime}}{s} \exp \left(-\frac{\left(s-s^{\prime}\right)^{2}}{2 s}\right),
$$

where $\inf _{\tilde{\eta}}$ denotes the infimum over all selectors and $\Psi$ is given in (21).

The proof is given in the Supplementary Material [8].

EXAMPLE 1. Let $\mathrm{f}_{a}$ be the Gaussian $\mathcal{N}\left(a, \sigma^{2}\right)$ density with some $\sigma^{2}>0$, and let $a_{0}<a_{1}$. For $f_{1}=\mathrm{f}_{a_{1}}$ and $f_{0}=\mathrm{f}_{a_{0}}$, the log-likelihood ratio

$$
\log \frac{f_{1}}{f_{0}}(X)=X \frac{a_{1}-a_{0}}{\sigma^{2}}-\frac{a_{1}^{2}-a_{0}^{2}}{2 \sigma^{2}}
$$


is increasing in $X$. By Theorem 3.3, the selector $\hat{\eta}$ on the class $\Theta_{d}^{+}\left(s, a_{0}, a_{1}\right)$ is a vector with components

$$
\hat{\eta}_{j}=I\left(X_{j} \geq t\left(a_{0}, a_{1}\right)\right), \quad j=1, \ldots, d,
$$

where

$$
t\left(a_{0}, a_{1}\right)=\frac{a_{1}+a_{0}}{2}+\frac{\sigma^{2} \log (d / s-1)}{a_{1}-a_{0}} .
$$

Note that for $a_{0}=0$ it coincides with the selector in (4) with $a=a_{1}$, which is minimax optimal on $\Theta_{d}^{+}\left(s, a_{1}\right)$. Moreover, the minimax risk only depends on $a_{0}$ and $a_{1}$ through the difference $\delta=a_{1}-a_{0}$ :

$$
\Psi=\Phi\left(-\frac{\delta}{2}+\frac{\sigma^{2} \log (d / s-1)}{\delta}\right)+\left(\frac{d}{s}-1\right) \Phi\left(-\frac{\delta}{2}+\frac{\sigma^{2} \log (d / s-1)}{\delta}\right) .
$$

EXAMPLE 2. Let $\mathbb{P}_{a}$ be the Bernoulli distribution $B(a)$ with parameter $a \in$ $(0,1)$, and $0<a_{0}<a_{1}<1$. Denoting by $f_{a}$ the density of $\mathbb{P}_{a}$ with respect to the counting measure we have, for $f_{1}=\mathrm{f}_{a_{1}}$ and $f_{0}=\mathrm{f}_{a_{0}}$,

$$
\log \frac{f_{1}}{f_{0}}(X)=X \log \left(\frac{a_{1}}{1-a_{1}} \frac{1-a_{0}}{a_{0}}\right)+\log \frac{1-a_{1}}{1-a_{0}}
$$

which is increasing in $X$ for $0<a_{0}<a_{1}<1$. The nearly minimax optimal selector $\hat{\eta}$ on the class $\Theta_{d}^{+}\left(s, a_{0}, a_{1}\right)$ is a vector with components $\hat{\eta}_{j}$ in (23) where the threshold $t\left(a_{0}, a_{1}\right)$ is given by

$$
t\left(a_{0}, a_{1}\right)=\frac{\log \left(\frac{d}{s}-1\right)-\log \frac{1-a_{1}}{1-a_{0}}}{\log \left(\frac{a_{1}}{1-a_{1}} \frac{1-a_{0}}{a_{0}}\right)} .
$$

Note that the nearly minimax selector $\hat{\eta}_{j}$ differs from the naive selector $\hat{\eta}_{j}^{n}=X_{j}$. Indeed since $X_{j} \in\{0,1\}$ we have $\hat{\eta}_{j}=1$ if either $X_{j}=1$ or $t\left(a_{0}, a_{1}\right) \leq 0$, and $\hat{\eta}_{j}=0$ if either $X_{j}=0$ or $t\left(a_{0}, a_{1}\right)>1$. The value $\Psi$ in the risk has the form

$$
\begin{aligned}
\Psi & =\mathbb{P}_{a_{1}}\left(X_{1}<t\left(a_{0}, a_{1}\right)\right)+\left(\frac{d}{s}-1\right) \mathbb{P}_{a_{0}}\left(X_{1} \geq t\left(a_{0}, a_{1}\right)\right) \\
& = \begin{cases}d / s-1, & t\left(a_{0}, a_{1}\right) \leq 0 \\
1-a_{1}+a_{0}(d / s-1), & 0<t\left(a_{0}, a_{1}\right)<1 \\
1, & t\left(a_{0}, a_{1}\right) \geq 1\end{cases}
\end{aligned}
$$

In the asymptotic regime when $d \rightarrow \infty$ and $s \rightarrow \infty$, the minimax risk is of order $s \Psi$ and can converge to 0 only when the parameters $d, s, a_{0}, a_{1}$ are kept such that $0<t\left(a_{0}, a_{1}\right)<1$, and in addition $\left(1-a_{1}\right) s \rightarrow 0, a_{0}(d-s) \rightarrow 0$. Thus, the risk can converge to 0 only when the Bernoulli probabilities $a_{1}$ and $a_{0}$ tend sufficiently fast to 1 and to 0 , respectively. 
ExAmPle 3. Let $\mathbb{P}_{a}$ be the Poisson distribution with parameter $a>0$, and let $a_{1}>a_{0}>0$. Denoting by $f_{a}$ the density of $\mathbb{P}_{a}$ with respect to the counting measure we have

$$
\log \frac{f_{1}}{f_{0}}(X)=X \log \left(\frac{a_{1}}{a_{0}}\right)-a_{1}+a_{0},
$$

which is increasing in $X$. The components of the nearly minimax optimal selector $\hat{\eta}$ are given by (23) with

$$
t\left(a_{0}, a_{1}\right)=\frac{\log (d / s-1)+a_{1}-a_{0}}{\log \left(a_{1} / a_{0}\right)} .
$$

Note that $t\left(a_{0}, a_{1}\right)>0$ as soon as $d / s \geq 2$ and $a_{1}>a_{0}>0$. The value of $\Psi$ in the risk has the form $\Psi=\mathbb{P}_{a_{1}}\left(X_{1}<t\left(a_{0}, a_{1}\right)\right)+(d / s-1) \mathbb{P}_{a_{0}}\left(X_{1} \geq t\left(a_{0}, a_{1}\right)\right)$.

3.3. Crowdsourcing with sparsity constraint. The problem of crowdsourcing with two classes is a clustering problem that can be formalized as follows; cf. [12]. Assume that $m$ workers provide class assignments for $d$ items. The class assignment $X_{i j}$ of the $i$ th worker for the $j$ th item is assumed to have a Bernoulli distribution $B\left(a_{i 0}\right)$ if the $j$ th item belongs to class 0 , and a Bernoulli distribution $B\left(a_{i 1}\right)$ if it belongs to class 1 . Here, $a_{i 0}, a_{i 1} \in(0,1)$ and $a_{i 0} \neq a_{i 1}$ for $i=1, \ldots, m$. The observations $\left(X_{i j}, i=1, \ldots, m, j=1, \ldots, d\right)$ are assumed to be jointly independent. Thus, each vector $X_{j}=\left(X_{1 j}, \ldots, X_{m j}\right)$ is distributed according to $P_{0}$ or to $P_{1}$ where each of these two measures is a product of Bernoulli measures, and $P_{0} \neq P_{1}$. We assume that there are at most $s$ vectors $X_{j}$ with distribution $P_{1}$, and the other vectors $X_{j}$ with distribution $P_{0}$. The aim is to recover the binary vector of class labels $\eta=\left(\eta_{1}, \ldots, \eta_{d}\right)$ based on the observations $\mathbf{X}=\left(X_{1}, \ldots, X_{d}\right)$. Here, $\eta_{j} \in\{0,1\}$ satisfies $\eta_{j}=k$ if the $j$ th item belongs to class $k \in\{0,1\}$. Thus, we are in the framework of Theorem 3.2 with a particular form of the log-likelihood ratio

$$
\log \frac{f_{1}}{f_{0}}\left(X_{j}\right)=\sum_{i=1}^{m}\left(X_{i j} \log \left(\frac{a_{i 1}}{1-a_{i 1}} \frac{1-a_{i 0}}{a_{i 0}}\right)+\log \frac{1-a_{i 1}}{1-a_{i 0}}\right),
$$

where $f_{k}$ is the density of $P_{k}, k \in\{0,1\}$. The following corollary is an immediate consequence of Theorem 3.2.

Corollary 3.1. Let $s<d, a_{i 0}, a_{i 1} \in(0,1)$ and $a_{i 0} \neq a_{i 1}$ for $i=1, \ldots, m$. Then the selector $\hat{\eta}$ in (19) with $\log \frac{f_{1}}{f_{0}}\left(X_{j}\right)$ defined in (24) satisfies Theorem 3.2.

For suitable combinations of parameters $d, s, a_{i 0}, a_{i 1}$, the exact asymptotic value of the minimax risk $\Psi$ can be further analyzed to obtain asymptotics of interest. Gao et al. [12] have studied a setting of crowdsourcing problem which is different from the one we consider here. They did not assume sparsity $s$, and instead of the class $\Theta_{d}\left(s, f_{0}, f_{1}\right)$ of at most $s$-sparse binary sequences, they considered the class of all possible binary sequences $\{0,1\}^{d}$. For this class, Gao et al. [12] analyzed specific asymptotics of the minimax risk $\inf _{\tilde{\eta}} \sup _{\eta \in\{0,1\}^{d}} d^{-1} \mathbf{E}|\tilde{\eta}-\eta|$ in large deviations perspective. 
4. Asymptotic analysis. Phase transitions. In this section, we conduct the asymptotic analysis of the problem of variable selection. The results are derived as corollaries of the minimax bounds of Section 2 . We will assume that $d \rightarrow \infty$ and that parameters $a=a_{d}$ and $s=s_{d}$ depend on $d$.

The first two asymptotic properties we study here are exact recovery and almost full recovery. We use this terminology following $[13,16]$ but we define these properties in a different way, as asymptotic minimax properties for classes of vectors $\theta$. The papers $[13,16]$ considered a Bayesian setup with random $\theta$ and studied a linear regression model with i.i.d. Gaussian regressors rather than the sequence model (1).

The study of exact recovery and almost full recovery will be done here only for the classes $\Theta_{d}\left(s_{d}, a_{d}\right)$. The corresponding results for the classes $\Theta_{d}^{+}\left(s_{d}, a_{d}\right)$ or $\Theta_{d}^{-}\left(s_{d}, a_{d}\right)$ are completely analogous. We do not state them here for the sake of brevity.

DEFINITION 4.1. Let $\left(\Theta_{d}\left(s_{d}, a_{d}\right)\right)_{d \geq 1}$ be a sequence of classes of sparse vectors:

- We say that exact recovery is possible for $\left(\Theta_{d}\left(s_{d}, a_{d}\right)\right)_{d \geq 1}$ if there exists a selector $\hat{\eta}$ such that

$$
\lim _{d \rightarrow \infty} \sup _{\theta \in \Theta_{d}\left(s_{d}, a_{d}\right)} \mathbf{E}_{\theta}|\hat{\eta}-\eta|=0
$$

In this case, we say that $\hat{\eta}$ achieves exact recovery.

- We say that almost full recovery is possible for $\left(\Theta_{d}\left(s_{d}, a_{d}\right)\right)_{d \geq 1}$ if there exists a selector $\hat{\eta}$ such that

$$
\lim _{d \rightarrow \infty} \sup _{\theta \in \Theta_{d}\left(s_{d}, a_{d}\right)} \frac{1}{s_{d}} \mathbf{E}_{\theta}|\hat{\eta}-\eta|=0 .
$$

In this case, we say that $\hat{\eta}$ achieves almost full recovery.

It is of interest to characterize the sequences $\left(s_{d}, a_{d}\right)_{d \geq 1}$, for which exact recovery and almost full recovery are possible. To describe the impossibility of exact or almost full recovery, we need the following definition.

DEFINITION 4.2. Let $\left(\Theta_{d}\left(s_{d}, a_{d}\right)\right)_{d \geq 1}$ be a sequence of classes of sparse vectors:

- We say that exact recovery is impossible for $\left(\Theta_{d}\left(s_{d}, a_{d}\right)\right)_{d \geq 1}$ if

$$
\liminf _{d \rightarrow \infty} \inf _{\tilde{\eta}} \sup _{\theta \in \Theta_{d}\left(s_{d}, a_{d}\right)} \mathbf{E}_{\theta}|\tilde{\eta}-\eta|>0
$$


- We say that almost full recovery is impossible for $\left(\Theta_{d}\left(s_{d}, a_{d}\right)\right)_{d \geq 1}$ if

$$
\liminf _{d \rightarrow \infty} \inf _{\tilde{\eta}} \sup _{\theta \in \Theta_{d}\left(s_{d}, a_{d}\right)} \frac{1}{s_{d}} \mathbf{E}_{\theta}|\tilde{\eta}-\eta|>0,
$$

where $\inf _{\tilde{\eta}}$ denotes the infimum over all selectors.

The following general characterization theorem is a straightforward corollary of the results of Section 2.

THEOREM 4.1. (i) Almost full recovery is possible for $\left(\Theta_{d}\left(s_{d}, a_{d}\right)\right)_{d \geq 1}$ if and only if $s_{d} \rightarrow \infty$ and

$$
\Psi_{+}\left(d, s_{d}, a_{d}\right) \rightarrow 0 \quad \text { as } d \rightarrow \infty .
$$

In this case, the selector $\hat{\eta}$ defined in (8) with threshold (5) achieves almost full recovery.

(ii) Exact recovery is possible for $\left(\Theta_{d}\left(s_{d}, a_{d}\right)\right)_{d \geq 1}$ if and only if

$$
s_{d} \Psi_{+}\left(d, s_{d}, a_{d}\right) \rightarrow 0 \quad \text { as } d \rightarrow \infty .
$$

In this case, the selector $\hat{\eta}$ defined in (8) with threshold (5) achieves exact recovery.

Although this theorem gives a complete solution to the problem, conditions (29) and (30) are not quite explicit. Intuitively, we would like to get a "phase transition" values $a_{d}^{*}$ such that exact (or almost full) recovery is possible for $a_{d}$ greater than $a_{d}^{*}$ and is impossible for $a_{d}$ smaller than $a_{d}^{*}$. Our aim now is to find such "phase transition" values. We first do it in the almost full recovery framework.

The following bounds for the tails of Gaussian distribution will be useful:

$$
\sqrt{\frac{2}{\pi}} \frac{e^{-y^{2} / 2}}{y+\sqrt{y^{2}+4}}<\frac{1}{\sqrt{2 \pi}} \int_{y}^{\infty} e^{-u^{2} / 2} d u \leq \sqrt{\frac{2}{\pi}} \frac{e^{-y^{2} / 2}}{y+\sqrt{y^{2}+8 / \pi}},
$$

for all $y \geq 0$. These bounds are an immediate consequence of formula 7.1.13 in [3] with $x=y / \sqrt{2}$.

Furthermore, we will need some nonasymptotic bounds for the expected Hamming loss that will play a key role in the subsequent asymptotic analysis. They are given in the next theorem.

THEOREM 4.2. Assume that $s<d / 2$.

(i) If

$$
a^{2} \geq \sigma^{2}(2 \log ((d-s) / s)+W) \quad \text { for some } W>0,
$$

then the selector $\hat{\eta}$ defined in (8) with threshold (5) satisfies

$$
\sup _{\theta \in \Theta_{d}(s, a)} \mathbf{E}_{\theta}|\hat{\eta}-\eta| \leq(2+\sqrt{2 \pi}) s \Phi(-\Delta),
$$


where $\Delta$ is defined by

$$
\Delta=\frac{W}{2 \sqrt{2 \log ((d-s) / s)+W}} .
$$

(ii) If $a>0$ is such that

$$
a^{2} \leq \sigma^{2}(2 \log ((d-s) / s)+W) \quad \text { for some } W>0,
$$

then, for any $s^{\prime}$ in $(0, s]$ we have

$$
\inf _{\tilde{\eta}} \sup _{\theta \in \Theta_{d}(s, a)} \mathbf{E}_{\theta}|\tilde{\eta}-\eta| \geq s^{\prime} \Phi(-\Delta)-4 s^{\prime} \exp \left(-\frac{\left(s-s^{\prime}\right)^{2}}{2 s}\right),
$$

where the infimum is taken over all selectors $\tilde{\eta}$ and $\Delta>0$ is defined in (34).

The proof is given in the Appendix.

The next theorem is an easy consequence of Theorem 4.2. It describes a "phase transition" for $a_{d}$ in the problem of almost full recovery.

TheOREM 4.3. Assume that $\lim _{\sup } \rightarrow \infty s_{d} / d<1 / 2$ :

(i) If, for all d large enough,

$$
a_{d}^{2} \geq \sigma^{2}\left(2 \log \left(\left(d-s_{d}\right) / s_{d}\right)+A_{d} \sqrt{2 \log \left(\left(d-s_{d}\right) / s_{d}\right)}\right)
$$

for an arbitrary sequence $A_{d} \rightarrow \infty$, as $d \rightarrow \infty$, then the selector $\hat{\eta}$ defined by (8) and (5) achieves almost full recovery:

$$
\lim _{d \rightarrow \infty} \sup _{\theta \in \Theta_{d}\left(s_{d}, a_{d}\right)} \frac{1}{s_{d}} \mathbf{E}_{\theta}|\hat{\eta}-\eta|=0 .
$$

(ii) Moreover, if there exists $A>0$ such that for all $s$ and $d$ large enough the reverse inequality holds:

$$
a_{d}^{2} \leq \sigma^{2}\left(2 \log \left(\left(d-s_{d}\right) / s_{d}\right)+A \sqrt{2 \log \left(\left(d-s_{d}\right) / s_{d}\right)}\right)
$$

then almost full recovery is impossible:

$$
\liminf _{d \rightarrow \infty} \inf _{\tilde{\eta}} \sup _{\theta \in \Theta_{d}\left(s_{d}, a_{d}\right)} \frac{1}{s_{d}} \mathbf{E}_{\theta}|\tilde{\eta}-\eta|>0 .
$$

Here, $\inf _{\tilde{\eta}}$ is the infimum over all selectors $\tilde{\eta}$.

The proof is given in the Appendix.

Inspection of the proof shows that the lower bound in Theorem 4.3 holds true for an arbitrary $s_{d} \geq 5$ (possibly fixed), if (37) is satisfied for some $A$ in $(0,1)$.

Under the sparsity assumption that

$$
s_{d} \rightarrow \infty, d / s_{d} \rightarrow \infty \quad \text { as } d \rightarrow \infty
$$


Theorem 4.3 shows that the "phase transition" for almost full recovery occurs at the value $a_{d}=a_{d}^{*}$, where

$$
a_{d}^{*}=\sigma \sqrt{2 \log \left(\left(d-s_{d}\right) / s_{d}\right)}(1+o(1)) .
$$

Furthermore, Theorem 4.3 details the behavior of the $o(1)$ term here.

We now state a corollary of Theorem 4.3 under simplified assumptions.

Corollary 4.1. Assume that (38) holds and set

$$
a_{d}=\sigma \sqrt{2(1+\delta) \log \left(d / s_{d}\right)} \quad \text { for some } \delta>0 .
$$

Then the selector $\hat{\eta}$ defined by (8) with threshold $t=\sigma \sqrt{2(1+\varepsilon(\delta)) \log \left(d / s_{d}\right)}$ where $\varepsilon(\delta)>0$ depends only on $\delta$, achieves almost full recovery.

In the particular case of $s_{d}=d^{1-\beta}(1+o(1))$ for some $\beta \in(0,1)$, condition (38) is satisfied. Then $\log \left(d / s_{d}\right)=\beta(1+o(1)) \log d$ and it follows from Corollary 4.1 that for $a_{d}=\sigma \sqrt{2 \beta(1+\delta) \log d}$ the selector with components $\hat{\eta}_{j}=I\left(\left|X_{j}\right|>\right.$ $\sigma \sqrt{2 \beta(1+\varepsilon) \log d})$ achieves almost full recovery. This is in agreement with the findings of $[13,16]$ where an analogous particular case of $s_{d}$ was considered for a different model and the Bayesian definition of almost full recovery.

We now turn to the problem of exact recovery. First, notice that if

$$
\limsup _{d \rightarrow \infty} s_{d}<\infty
$$

the properties of exact recovery and almost full recovery are equivalent. Therefore, it suffices to consider exact recovery only when $s_{d} \rightarrow \infty$ as $d \rightarrow \infty$. Under this assumption, the "phase transition" for $a_{d}$ in the problem of exact recovery is described in the next theorem.

THEOREM 4.4. Assume that $\lim _{d \rightarrow \infty} s_{d}=\infty$ and $\lim \sup _{d \rightarrow \infty} s_{d} / d<1 / 2$.

(i) If

$$
a_{d}^{2} \geq \sigma^{2}\left(2 \log \left(\left(d-s_{d}\right) / s_{d}\right)+W_{d}\right)
$$

for all d large enough, where the sequence $W_{d}$ is such that

$$
\liminf _{d \rightarrow \infty} \frac{W_{d}}{4\left(\log \left(s_{d}\right)+\sqrt{\log \left(s_{d}\right) \log \left(d-s_{d}\right)}\right)} \geq 1,
$$

then the selector $\hat{\eta}$ defined by (8) and (5) achieves exact recovery:

$$
\lim _{d \rightarrow \infty} \sup _{\theta \in \Theta_{d}\left(s_{d}, a_{d}\right)} \mathbf{E}_{\theta}|\hat{\eta}-\eta|=0 .
$$


(ii) If the complementary condition holds,

$$
a_{d}^{2} \leq \sigma^{2}\left(2 \log \left(\left(d-s_{d}\right) / s_{d}\right)+W_{d}\right)
$$

for all d large enough, where the sequence $W_{d}$ is such that

$$
\limsup _{d \rightarrow \infty} \frac{W_{d}}{4\left(\log \left(s_{d}\right)+\sqrt{\log \left(s_{d}\right) \log \left(d-s_{d}\right)}\right)}<1,
$$

then exact recovery is impossible, and moreover we have

$$
\lim _{d \rightarrow \infty} \inf _{\tilde{\eta}} \sup _{\theta \in \Theta_{d}\left(s_{d}, a_{d}\right)} \mathbf{E}_{\theta}|\tilde{\eta}-\eta|=\infty .
$$

Here, $\inf _{\tilde{\eta}}$ is the infimum over all selectors $\tilde{\eta}$.

The proof is given in the Appendix.

Some remarks are in order here. First of all, Theorem 4.4 shows that the "phase transition" for exact recovery occurs at $W_{d}=4\left(\log \left(s_{d}\right)+\sqrt{\log \left(s_{d}\right) \log \left(d-s_{d}\right)}\right)$, which corresponds to the critical value $a_{d}=a_{d}^{*}$ of the form

$$
a_{d}^{*}=\sigma\left(\sqrt{2 \log \left(d-s_{d}\right)}+\sqrt{2 \log s_{d}}\right) .
$$

This value is greater than the critical value $a_{d}^{*}$ for almost full recovery [cf. (39)], which is intuitively quite clear. The optimal threshold (5) corresponding to (43) has a simple form:

$$
t_{d}^{*}=\frac{a_{d}^{*}}{2}+\frac{\sigma^{2}}{a_{d}^{*}} \log \left(\frac{d}{s_{d}}-1\right)=\sigma \sqrt{2 \log \left(d-s_{d}\right)} .
$$

For example, if $s_{d}=d^{1-\beta}(1+o(1))$ for some $\beta \in(0,1)$, then $a_{d}^{*} \sim \sigma(1+$ $\sqrt{1-\beta}) \sqrt{2 \log d}$. In this particular case, Theorem 4.4 implies that if $a_{d}=\sigma(1+$ $\sqrt{1-\beta}) \sqrt{2(1+\delta) \log d}$ for some $\delta>0$, then exact recovery is possible and the selector with threshold $t=\sigma \sqrt{2(1+\varepsilon) \log d}$ for some $\varepsilon>0$ achieves exact recovery. This is in agreement with the results of $[13,16]$ where an analogous particular case of $s_{d}$ was considered for a different model and the Bayesian definition of exact recovery. For our model, even a sharper result is true; namely, a simple universal threshold $t=\sigma \sqrt{2 \log d}$ guarantees exact recovery adaptively in the parameters $a$ and $s$. Intuitively, this is suggested by the form of $t_{d}^{*}$. The precise statement is given in Theorem 5.1 below.

Finally, we state an asymptotic corollary of Theorem 2.6 showing that the selector $\hat{\eta}$ considered above is sharp in the asymptotically minimax sense with respect to the risk defined as the probability of wrong recovery.

THEOREM 4.5. Assume that exact recovery is possible for the classes $\left(\Theta_{d}\left(s_{d}, a_{d}\right)\right)_{d \geq 1}$ and $\left(\Theta_{d}^{+}\left(s_{d}, a_{d}\right)\right)_{d \geq 1}$, that is, condition (30) holds. Then, for the 
selectors $\hat{\eta}$ and $\hat{\eta}^{+}$defined by (8), (4) and (5), and for the selector $\bar{\eta}$ defined by (12) and (13), we have

$$
\begin{aligned}
& \lim _{d \rightarrow \infty} \sup _{\theta \in \Theta_{d}^{+}\left(s_{d}, a_{d}\right)} \frac{\mathbf{P}_{\theta}\left(S_{\hat{\eta}^{+}} \neq S(\theta)\right)}{s_{d} \Psi_{+}\left(d, s_{d}, a_{d}\right)}=\lim _{d \rightarrow \infty} \inf _{\tilde{\eta} \in \mathcal{T}} \sup _{\theta \in \Theta_{d}^{+}\left(s_{d}, a_{d}\right)} \frac{\mathbf{P}_{\theta}\left(S_{\tilde{\eta}} \neq S(\theta)\right)}{s_{d} \Psi_{+}\left(d, s_{d}, a_{d}\right)}=1, \\
& \lim _{d \rightarrow \infty} \sup _{\theta \in \Theta_{d}\left(s_{d}, a_{d}\right)} \frac{\mathbf{P}_{\theta}\left(S_{\bar{\eta}} \neq S(\theta)\right)}{s_{d} \bar{\Psi}\left(d, s_{d}, a_{d}\right)}=\lim _{d \rightarrow \infty} \inf _{\tilde{\eta} \in \mathcal{T}} \sup _{\theta \in \Theta_{d}\left(s_{d}, a_{d}\right)} \frac{\mathbf{P}_{\theta}\left(S_{\tilde{\eta}} \neq S(\theta)\right)}{s_{d} \bar{\Psi}\left(d, s_{d}, a_{d}\right)}=1
\end{aligned}
$$

and

$$
\limsup _{d \rightarrow \infty} \sup _{\theta \in \Theta_{d}\left(s_{d}, a_{d}\right)} \frac{\mathbf{P}_{\theta}\left(S_{\hat{\eta}} \neq S(\theta)\right)}{s_{d} \Psi_{+}\left(d, s_{d}, a_{d}\right)} \leq 2 .
$$

Note that the threshold (5) depends on the parameters $s$ and $a$, so that the selectors considered in all the results above are not adaptive. In the next section, we propose adaptive selectors that achieve almost full recovery and exact recovery without the knowledge of $s$ and $a$.

REMARK 4.1. Another procedure of variable selection is the exhaustive search estimator of the support $S(\theta)$ defined as

$$
\tilde{S}=\underset{C \subseteq\{1, \ldots, d\}:|C|=s}{\operatorname{argmax}} \sum_{j \in C} X_{j} .
$$

This estimator was studied by Butucea et al. [7]. The selection procedure can be equivalently stated as choosing the indices $j$ corresponding to $s$ largest order statistics of the sample $\left(X_{1}, \ldots, X_{d}\right)$. In [7], Theorem 2.5, it was shown that, on the class $\Theta_{d}^{+}\left(s_{d}, a_{d}\right)$, the probability of wrong recovery $\mathbf{P}_{\theta}(\tilde{S} \neq S(\theta))$ tends to 0 as $d \rightarrow \infty$ under a stronger condition on $\left(s_{d}, a_{d}\right)$ than (30). The rate of this convergence was not analyzed there. If we denote by $\eta_{\tilde{S}}$ the selector with components $I(j \in \tilde{S})$ for $j$ from 1 to $d$, it can be proved that $\mathbf{E}\left|\eta_{\tilde{S}}-\eta\right| \leq 2 \mathbf{E}\left|\hat{\eta}^{+}-\eta\right|$, and thus the risk of $\eta_{\tilde{S}}$ is within at least a factor 2 of the minimax risk over the class $\Theta_{d}^{+}(s, a)$.

5. Adaptive selectors. In this section, we consider the asymptotic setup as in Section 4 and construct the selectors that provide almost full and exact recovery adaptively, that is, without the knowledge of $a$ and $s$.

As discussed in Section 4, the issue of adaptation for exact recovery is almost trivial. Indeed, the expressions for minimal value $a_{d}^{*}$, for which exact recovery is possible [cf. (43)], and for the corresponding optimal threshold $t_{d}^{*}$ suggest that taking a selector with the universal threshold $t=\sigma \sqrt{2 \log d}$ is enough to achieve exact recovery simultaneously for all values $\left(a_{d}, s_{d}\right)$, for which the exact recovery is possible. This point is formalized in the next theorem. 
THEOREM 5.1. Assume that $s_{d} \rightarrow \infty$ as $d \rightarrow \infty$ and that $\lim _{\sup } \sup _{d \rightarrow \infty} s_{d} / d<$ $1 / 2$. Let the sequence $\left(a_{d}\right)_{d \geq 1}$ be above the phase transition level for exact recovery, that is, $a_{d} \geq a_{d}^{*}$ for all $d$, where $a_{d}^{*}$ is defined in (43). Then the selector $\hat{\eta}$ defined by (8) with threshold $t=\sigma \sqrt{2 \log d}$ achieves exact recovery.

The proof of this theorem is given in the Appendix.

We now turn to the problem of adaption for almost full recovery. Ideally, we would like to construct a selector that achieves almost full recovery for all sequences $\left(s_{d}, a_{d}\right)_{d \geq 1}$ for which almost full recovery is possible. We have seen in Section 4 that this includes a much broader range of values than in case of exact recovery. Thus, using the adaptive selector of Theorem 5.1 for almost full recovery does not give a satisfactory result, and we have to take a different approach.

Following Section 4, we will use the notation

$$
a_{0}(s, A) \triangleq \sigma(2 \log ((d-s) / s)+A \sqrt{\log ((d-s) / s)})^{1 / 2} .
$$

As shown in Section 4, it makes sense to consider the classes $\Theta_{d}(s, a)$ only when $a \geq a_{0}(s, A)$ with some $A>0$, since for other values of $a$ almost full recovery is impossible. Only such classes will be studied below.

In the asymptotic setup of Section 4 , we have used the assumption that $d / s_{d} \rightarrow$ $\infty$ (the sparsity assumption), which is now transformed into the condition

$$
s_{d} \in \mathcal{S}_{d} \triangleq\left\{1,2, \ldots, s_{d}^{*}\right\}
$$

$$
\text { where } s_{d}^{*} \text { is an integer such that } \frac{d}{s_{d}^{*}} \rightarrow \infty \text { as } d \rightarrow \infty \text {. }
$$

Assuming $s_{d}$ to be known, we have shown in Section 4 that almost full recovery is achievable for all $a \geq a_{0}\left(s_{d}, A_{d}\right)$, where $A_{d}$ tends to infinity as $d \rightarrow \infty$. The rate of growth of $A_{d}$ was allowed to be arbitrarily slow there; cf. Theorem 4.3. However, for adaptive estimation considered in this section we will need the following mild assumption on the growth of $A_{d}$ :

$$
A_{d} \geq c_{0}\left(\log \log \left(\frac{d}{s_{d}^{*}}-1\right)\right)^{1 / 2}
$$

where $c_{0}>0$ is an absolute constant. In what follows, we will assume that $s_{d}^{*} \leq$ $d / 4$, so that the right-hand side of (45) is well defined.

Consider a grid of points $\left\{g_{1}, \ldots, g_{M}\right\}$ on $\mathcal{S}_{d}$, where $g_{j}=2^{j-1}$ and $M$ is the maximal integer such that $g_{M} \leq s_{d}^{*}$. For each $g_{m}, m=1, \ldots, M$, we define a selector:

$$
\hat{\eta}\left(g_{m}\right)=\left(\hat{\eta}_{j}\left(g_{m}\right)\right)_{j=1, \ldots, d} \triangleq\left(I\left(\left|X_{j}\right| \geq w\left(g_{m}\right)\right)\right)_{j=1, \ldots, d},
$$


where

$$
w(s)=\sigma \sqrt{2 \log \left(\frac{d}{s}-1\right)} .
$$

Note that $w(s)$ is monotonically decreasing. We now choose the "best" index $m$, for which $g_{m}$ is near the true (but unknown) value of $s$, by the following datadriven procedure:

$$
\begin{aligned}
\hat{m}= & \min \{m \in\{2, \ldots, M\}: \\
& \left.\sum_{j=1}^{d} I\left(w\left(g_{k}\right) \leq\left|X_{j}\right|<w\left(g_{k-1}\right)\right) \leq \tau g_{k} \text { for all } k \geq m+1\right\},
\end{aligned}
$$

where

$$
\tau=\left(\log \left(d / s_{d}^{*}-1\right)\right)^{-\frac{1}{7}}
$$

and we set $\hat{m}=M$ if the set in (46) is empty. Finally, we define an adaptive selector as

$$
\hat{\eta}^{\mathrm{ad}}=\hat{\eta}\left(g_{\hat{m}}\right)
$$

This adaptive procedure is quite natural in the sense that it can be related to the Lepski method or to wavelet thresholding that are widely used for adaptive estimation. Indeed, as in wavelet methods, we consider dyadic blocks determined by the grid points $g_{j}$. The value $\sum_{j=1}^{d} I\left(w\left(g_{k}\right) \leq\left|X_{j}\right|<w\left(g_{k-1}\right)\right)$ is the number of observations within the $k$ th block. If this number is too small (below a suitably chosen threshold), we decide that the block corresponds to pure noise and it is rejected; in other words, this $k$ is not considered as a good candidate for $\hat{m}$. This argument is analogous to wavelet thersholding. We start from the largest $k$ [equivalently, smallest $w\left(g_{k}\right)$ ] and perform this procedure until we find the first block, which is not rejected. The corresponding value $k$ determines our choice of $\hat{m}$ as defined in (46).

THEOREM 5.2. Let $c_{0} \geq 16$. Then the selector $\hat{\eta}^{\mathrm{ad}}$ adaptively achieves almost full recovery in the following sense:

$$
\lim _{d \rightarrow \infty} \sup _{\theta \in \Theta_{d}\left(s_{d}, a_{d}\right)} \frac{1}{s_{d}} \mathbf{E}_{\theta}\left|\hat{\eta}^{\mathrm{ad}}-\eta\right|=0
$$

for all sequences $\left(s_{d}, a_{d}\right)_{d \geq 1}$ such that (44) holds and $a_{d} \geq a_{0}\left(s_{d}, A_{d}\right)$, where $A_{d}$ satisfies (45). 
REMARK 5.1. Another family of variable selection methods originates from the theory of multiple testing [1, 2]. These are, for example, the BenjaminiHochberg, Benjamini-Yekutieli or SLOPE procedures. We refer to [6] for a recent overview and comparison of these techniques. They have the same structure as the exhaustive search procedure in that they keep only the largest order statistics. The difference is that the value $s$ (which is usually not known in practice) is replaced by an estimator $\hat{s}$ obtained from comparing the $i$ th order statistic of $\left(\left|X_{1}\right|, \ldots,\left|X_{d}\right|\right)$ with a suitable normal quantile depending on $i$. The analysis of these methods in the literature is focused on the evaluation of false discovery rate (FDR). Asymptotic power calculations for the Benjamini-Hochberg procedure are given in [4]. To the best of our knowledge, the behavior of the risk $\mathbf{P}_{\theta}(\tilde{S} \neq S(\theta))$ and of the Hamming risk, even in a simple consistency perspective, was not studied.

REMARK 5.2. In this paper, the variance $\sigma$ was supposed to be known. Extension to the case of unknown $\sigma$ can be treated as described, for example, in [10]. Namely, we replace $\sigma$ in the definition of the threshold $w(s)$ by a statistic $\hat{\sigma}$ defined in [10], Section 3. As shown in [10], Proposition 1, this statistic is such that $\sigma \leq \hat{\sigma} \leq C^{\prime} \sigma$ with high probability provided that $s \leq d / 2$, and $d \geq d_{0}$ for some absolute constants $C^{\prime}>1, d_{0} \geq 1$. Then, replacing $\sigma$ by $\hat{\sigma}$ in the expression for $w(s)$, one can show that Theorem 5.2 remains valid with this choice of $w(s)$ independent of $\sigma$, up to a change in numerical constants in the definition of the adaptive procedure. With this modification, we obtain a procedure which is completely data-driven and enjoys the property of almost full recovery under the mild conditions given in Theorem 5.2. The same modification can be done in Theorem 5.1. Namely, under the assumptions of Theorem 5.1 and $a_{d} \geq c^{\prime} a_{d}^{*}$, where $c^{\prime} \geq 1$ is a numerical constant, the selector $\hat{\eta}$ defined by (8) with threshold $t=\hat{\sigma} \sqrt{2 \log d}$ achieves exact recovery when $\sigma$ is unknown.

REMARK 5.3. In this section, the problem of adaptive variable selection was considered only for the classes $\Theta_{d}\left(s_{d}, a_{d}\right)$. The corresponding results for classes $\Theta_{d}^{+}\left(s_{d}, a_{d}\right)$ and $\Theta_{d}^{-}\left(s_{d}, a_{d}\right)$ are completely analogous. We do not state them here for the sake of brevity.

\section{APPENDIX}

Proof of TheOrem 2.3. We have, for any $t>0$,

$$
\begin{aligned}
|\hat{\eta}-\eta| & =\sum_{j: \eta_{j}=0} \hat{\eta}_{j}+\sum_{j: \eta_{j}=1}\left(1-\hat{\eta}_{j}\right) \\
& =\sum_{j: \eta_{j}=0} I\left(\left|\sigma \xi_{j}\right| \geq t\right)+\sum_{j: \eta_{j}=1} I\left(\left|\sigma \xi_{j}+\theta_{j}\right|<t\right) .
\end{aligned}
$$


Now, for any $\theta \in \Theta_{d}(s, a)$ and any $t>0$,

$$
\begin{aligned}
\mathbf{E}\left(I\left(\left|\sigma \xi_{j}+\theta_{j}\right|<t\right)\right) & \leq \mathbf{P}\left(\left|\theta_{j}\right|-\left|\sigma \xi_{j}\right|<t\right) \leq \mathbf{P}(|\xi|>(a-t) / \sigma) \\
& =\mathbf{P}\left(|\xi|>(a-t)_{+} / \sigma\right)
\end{aligned}
$$

where $\xi$ denotes a standard Gaussian random variable. Thus, for any $\theta \in \Theta_{d}(s, a)$,

(48) $\frac{1}{s} \mathbf{E}_{\theta}|\hat{\eta}-\eta| \leq \frac{d-|S|}{s} \mathbf{P}\left(|\xi| \geq \frac{t}{\sigma}\right)+\frac{|S|}{s} \mathbf{P}\left(|\xi|>\frac{(a-t)_{+}}{\sigma}\right) \leq 2 \Psi(d, s, a)$.

Indeed, for $t$ defined in (5), $t \geq(a-t)_{+}$given that $s \leq d / 2$. Here and in the sequel, $|S|$ denotes the cardinality of $S=S(\theta)$.

Proof of TheOREM 2.1. Arguing as in the proof of Theorem 2.3, we obtain

$$
\left|\hat{\eta}^{+}-\eta\right|=\sum_{j: \eta_{j}=0} I\left(\xi_{j} \geq t\right)+\sum_{j: \eta_{j}=1} I\left(\sigma \xi_{j}+\theta_{j}<t\right)
$$

and $\mathbf{E}\left(I\left(\sigma \xi_{j}+\theta_{j}<t\right)\right) \leq \mathbf{P}(\xi<(t-a) / \sigma)$. Thus, for any $\theta \in \Theta_{d}^{+}(s, a)$,

$$
\frac{1}{s} \mathbf{E}_{\theta}\left|\hat{\eta}^{+}-\eta\right| \leq \frac{d-|S|}{s} \mathbf{P}(\xi \geq t / \sigma)+\frac{|S|}{s} \mathbf{P}(\xi<(t-a) / \sigma) \leq \Psi_{+}(d, s, a),
$$

by the monotonicity of $\Phi$ and the condition $s \leq d / 2$.

ProOF OF THEOREM 2.2. We prove here the first inequality of Theorem 2.2. Since $\tilde{\eta}_{j}$ depends only on $X_{j}$,

$$
\mathbf{E}_{\theta}|\tilde{\eta}-\eta|=\sum_{j=1}^{d} \mathbf{E}_{j, \theta_{j}}\left|\tilde{\eta}_{j}-\eta_{j}\right|
$$

where $\mathbf{E}_{j, \theta_{j}}$ is the expectation with respect to the distribution of $X_{j}$.

Let $\Theta^{\prime}$ be the set of all $\theta$ in $\Theta_{d}^{+}(s, a)$ such that $s$ components $\theta_{j}$ of $\theta$ are equal to $a$ and the remaining $d-s$ components are 0 . Denote by $\left|\Theta^{\prime}\right|=\left(\begin{array}{l}d \\ s\end{array}\right)$ the cardinality 
of $\Theta^{\prime}$. Then, for any $\tilde{\eta} \in \mathcal{T}$ we have

$$
\begin{aligned}
& \sup _{\theta \in \Theta_{d}^{+}(s, a)} \quad \frac{1}{s} \mathbf{E}_{\theta}|\tilde{\eta}-\eta| \\
& \quad \geq \frac{1}{s\left|\Theta^{\prime}\right|} \sum_{\theta \in \Theta^{\prime}} \sum_{j=1}^{d} \mathbf{E}_{j, \theta_{j}}\left|\tilde{\eta}_{j}-\eta_{j}\right| \\
& =\frac{1}{s\left|\Theta^{\prime}\right|} \sum_{j=1}^{d}\left(\sum_{\theta \in \Theta^{\prime}: \theta_{j}=0} \mathbf{E}_{j, 0}\left(\tilde{\eta}_{j}\right)+\sum_{\theta \in \Theta^{\prime}: \theta_{j}=a} \mathbf{E}_{j, a}\left(1-\tilde{\eta}_{j}\right)\right) \\
& =\frac{1}{s} \sum_{j=1}^{d}\left(\left(1-\frac{s}{d}\right) \mathbf{E}_{j, 0}\left(\tilde{\eta}_{j}\right)+\frac{s}{d} \mathbf{E}_{j, a}\left(1-\tilde{\eta}_{j}\right)\right) \\
& \geq \frac{d}{s} \inf _{T \in[0,1]}\left(\left(1-\frac{s}{d}\right) \mathbb{E}_{0}(T)+\frac{s}{d} \mathbb{E}_{a}(1-T)\right),
\end{aligned}
$$

where we have used that $\left|\left\{\theta \in \Theta^{\prime}: \theta_{j}=a\right\}\right|=\left(\begin{array}{c}d-1 \\ s-1\end{array}\right)=s\left|\Theta^{\prime}\right| / d$. In the last line of display (50), $\mathbb{E}_{u}$ is understood as the expectation with respect to the distribution of $X=u+\sigma \xi$, where $\xi \sim \mathcal{N}(0,1)$ and $\inf _{T \in[0,1]}$ denotes the infimum over all $[0,1]$-valued statistics $T(X)$. Set

$$
L^{*}=\inf _{T \in[0,1]}\left(\left(1-\frac{s}{d}\right) \mathbb{E}_{0}(T)+\frac{s}{d} \mathbb{E}_{a}(1-T)\right)
$$

By the Bayesian version of the Neyman-Pearson lemma, the infimum here is attained for $T=T^{*}$ given by

$$
T^{*}(X)=I\left(\frac{(s / d) \varphi_{\sigma}(X-a)}{(1-s / d) \varphi_{\sigma}(X)}>1\right)
$$

where $\varphi_{\sigma}(\cdot)$ is the density of an $\mathcal{N}\left(0, \sigma^{2}\right)$ distribution. Thus,

$$
L^{*}=\left(1-\frac{s}{d}\right) \mathbf{P}\left(\frac{\varphi_{\sigma}(\sigma \xi-a)}{\varphi_{\sigma}(\sigma \xi)}>\frac{d}{s}-1\right)+\frac{s}{d} \mathbf{P}\left(\frac{\varphi_{\sigma}(\sigma \xi)}{\varphi_{\sigma}(\sigma \xi+a)} \leq \frac{d}{s}-1\right) .
$$

Combining this with (49) and (50), we get

$$
\begin{aligned}
\inf _{\tilde{\eta}} \sup _{\theta \in \Theta_{d}^{+}(s, a)} \frac{1}{s} \mathbf{E}_{\theta}|\tilde{\eta}-\eta| \\
\geq \\
\geq\left(\frac{d}{s}-1\right) \mathbf{P}\left(\exp \left(\frac{a \xi}{\sigma}-\frac{a^{2}}{2 \sigma^{2}}\right)>\frac{d}{s}-1\right) \\
\quad+\mathbf{P}\left(\exp \left(\frac{a \xi}{\sigma}+\frac{a^{2}}{2 \sigma^{2}}\right) \leq \frac{d}{s}-1\right)
\end{aligned}
$$




$$
\begin{aligned}
= & \left(\frac{d}{s}-1\right) \mathbf{P}\left(\xi>\frac{a}{2 \sigma}+\frac{\sigma}{a} \log \left(\frac{d}{s}-1\right)\right) \\
& +\mathbf{P}\left(\xi \leq-\frac{a}{2 \sigma}+\frac{\sigma}{a} \log \left(\frac{d}{s}-1\right)\right) \\
= & \Psi_{+}(d, s, a) .
\end{aligned}
$$

Proof of TheOREM 2.4. For any $\theta \in \Theta_{d}(s, a)$, we have

$$
\begin{aligned}
\mathbf{E}_{\theta}|\bar{\eta}-\eta|= & \sum_{j: \theta_{j}=0} \mathbf{P}_{j, 0}\left(\bar{\eta}_{j}=1\right)+\sum_{j: \theta_{j} \geq a} \mathbf{P}_{j, \theta_{j}}\left(\bar{\eta}_{j}=0\right) \\
& +\sum_{j: \theta_{j} \leq-a} \mathbf{P}_{j, \theta_{j}}\left(\bar{\eta}_{j}=0\right) \\
\leq & d \mathbf{P}\left(e^{-\frac{a^{2}}{2 \sigma^{2}}} \cosh \left(\frac{a \xi}{\sigma}\right)>\frac{d}{s}-1\right) \\
& +\sum_{j: \theta_{j} \geq a} \mathbf{P}_{j, \theta_{j}}\left(\bar{\eta}_{j}=0\right)+\sum_{j: \theta_{j} \leq-a} \mathbf{P}_{j, \theta_{j}}\left(\bar{\eta}_{j}=0\right),
\end{aligned}
$$

where $\mathbf{P}_{j, \theta_{j}}$ denotes the distribution of $X_{j}$, and $\xi$ is a standard Gaussian random variable. We now bound from above the probabilities $\mathbf{P}_{j, \theta_{j}}\left(\bar{\eta}_{j}=0\right)$. Introduce the notation

$$
g(x)=\cosh \left(\frac{(x+\sigma \xi) a}{\sigma^{2}}\right) \quad \forall x \in \mathbb{R},
$$

and

$$
u=\exp \left(\frac{a^{2}}{2 \sigma^{2}}+\log \left(\frac{d}{s}-1\right)\right)
$$

We have

$$
\mathbf{P}_{j, \theta_{j}}\left(\bar{\eta}_{j}=0\right)=\mathbf{P}\left(g\left(\theta_{j}\right)<u\right)=\mathbf{P}\left(-b-\theta_{j}<\sigma \xi<b-\theta_{j}\right),
$$

where $b=\left(\sigma^{2} / a\right) \operatorname{arccosh}(u)>0$. It is easy to check that the function $x \mapsto$ $\mathbf{P}(-b-x<\sigma \xi<b-x)$ is monotonically decreasing on $[0, \infty)$. Therefore, the maximum of $\mathbf{P}\left(-b-\theta_{j}<\sigma \xi<b-\theta_{j}\right)$ over $\theta_{j} \geq a$ is attained at $\theta_{j}=a$. Thus, for any $\theta_{j} \geq a$ we have

$$
\mathbf{P}_{j, \theta_{j}}\left(\bar{\eta}_{j}=0\right) \leq \mathbf{P}(g(a)<u)=\mathbf{P}\left(e^{-\frac{a^{2}}{2 \sigma^{2}}} \cosh \left(\frac{(a+\sigma \xi) a}{\sigma^{2}}\right)<\frac{d}{s}-1\right) .
$$

Analogously, for any $\theta_{j} \leq-a$,

$$
\begin{aligned}
\mathbf{P}_{j, \theta_{j}}\left(\bar{\eta}_{j}=0\right) & \leq \mathbf{P}\left(e^{-\frac{a^{2}}{2 \sigma^{2}}} \cosh \left(\frac{(-a+\sigma \xi) a}{\sigma^{2}}\right)<\frac{d}{s}-1\right) \\
& =\mathbf{P}\left(e^{-\frac{a^{2}}{2 \sigma^{2}}} \cosh \left(\frac{(a+\sigma \xi) a}{\sigma^{2}}\right)<\frac{d}{s}-1\right),
\end{aligned}
$$


where the last equality follows from the fact that $\xi$ has the same distribution as $-\xi$ and cosh is an even function. Combining (51)-(53) proves the theorem.

Proof of Theorem 2.5. We follow the lines of the proof of Theorem 2.2 with suitable modifications.

Let $\Theta^{+}$and $\Theta^{-}$be the sets of all $\theta$ in $\Theta_{d}(s, a)$ such that $d-s$ components $\theta_{j}$ of $\theta$ are equal to 0 and the remaining $s$ components are equal to $a$ (for $\theta \in \Theta^{+}$) or to $-a$ (for $\theta \in \Theta^{-}$). For any $\tilde{\eta} \in \mathcal{T}$, we have

$$
\begin{aligned}
& \sup _{\theta \in \Theta_{d}(s, a)} \sum_{j=1}^{d} \mathbf{E}_{j, \theta_{j}}\left|\tilde{\eta}_{j}-\eta_{j}\right| \\
& \geq \frac{1}{2}\left\{\sup _{\theta \in \Theta^{+}} \sum_{j=1}^{d} \mathbf{E}_{j, \theta_{j}}\left|\tilde{\eta}_{j}-\eta_{j}\right|+\sup _{\theta \in \Theta^{-}} \sum_{j=1}^{d} \mathbf{E}_{j, \theta_{j}}\left|\tilde{\eta}_{j}-\eta_{j}\right|\right\} .
\end{aligned}
$$

As shown in the proof of Theorem 2.2, for any $\tilde{\eta} \in \mathcal{T}$,

$$
\sup _{\theta \in \Theta^{+}} \sum_{j=1}^{d} \mathbf{E}_{j, \theta_{j}}\left|\tilde{\eta}_{j}-\eta_{j}\right| \geq \sum_{j=1}^{d}\left(\left(1-\frac{s}{d}\right) \mathbf{E}_{j, 0}\left(\tilde{\eta}_{j}\right)+\frac{s}{d} \mathbf{E}_{j, a}\left(1-\tilde{\eta}_{j}\right)\right) .
$$

Analogously,

$$
\sup _{\theta \in \Theta^{-}} \sum_{j=1}^{d} \mathbf{E}_{j, \theta_{j}}\left|\tilde{\eta}_{j}-\eta_{j}\right| \geq \sum_{j=1}^{d}\left(\left(1-\frac{s}{d}\right) \mathbf{E}_{j, 0}\left(\tilde{\eta}_{j}\right)+\frac{s}{d} \mathbf{E}_{j,-a}\left(1-\tilde{\eta}_{j}\right)\right) .
$$

From the last three displays, we obtain

$$
\sup _{\theta \in \Theta_{d}(s, a)} \sum_{j=1}^{d} \mathbf{E}_{j, \theta_{j}}\left|\tilde{\eta}_{j}-\eta_{j}\right| \geq \sum_{j=1}^{d}\left(\left(1-\frac{s}{d}\right) \mathbf{E}_{j, 0}\left(\tilde{\eta}_{j}\right)+\frac{s}{d} \overline{\mathbf{E}}_{j}\left(1-\tilde{\eta}_{j}\right)\right),
$$

where $\overline{\mathbf{E}}_{j}$ is the expectation with respect to the measure $\overline{\mathbf{P}}_{j}=\left(\mathbf{P}_{j, a}+\mathbf{P}_{j,-a}\right) / 2$. It follows that

$$
\sup _{\theta \in \Theta_{d}(s, a)} \sum_{j=1}^{d} \mathbf{E}_{j, \theta_{j}}\left|\tilde{\eta}_{j}-\eta_{j}\right| \geq \inf _{T \in[0,1]}\left((d-s) \mathbb{E}_{0}(T)+s \overline{\mathbb{E}}(1-T)\right) .
$$

Here, $\mathbb{E}_{0}$ denotes the expectation with respect to the distribution of $X$ with density $\varphi_{\sigma}(\cdot), \overline{\mathbb{E}}$ is the expectation with respect to the distribution of $X$ with mixture density $\bar{\varphi}_{\sigma}(\cdot)=\left(\varphi_{\sigma}(\cdot+a)+\varphi_{\sigma}(\cdot-a)\right) / 2$, and $\inf _{T \in[0,1]}$ denotes the infimum over all $[0,1]$-valued statistics $T(X)$. Recall that we denote by $\varphi_{\sigma}(\cdot)$ is the density of $\mathcal{N}\left(0, \sigma^{2}\right)$ distribution. Set

$$
\tilde{L}=\inf _{T \in[0,1]}\left(\left(1-\frac{s}{d}\right) \mathbb{E}_{0}(T)+\frac{s}{d} \overline{\mathbb{E}}(1-T)\right) .
$$


By the Bayesian version of the Neyman-Pearson lemma, the infimum here is attained for $T=\tilde{T}$ given by

$$
\tilde{T}(X)=I\left(\frac{(s / d) \bar{\varphi}_{\sigma}(X)}{(1-s / d) \varphi_{\sigma}(X)}>1\right) .
$$

Thus,

$$
\begin{aligned}
\tilde{L}= & \left(1-\frac{s}{d}\right) \mathbf{P}\left(\frac{\bar{\varphi}_{\sigma}(\sigma \xi)}{\varphi_{\sigma}(\sigma \xi)}>\frac{d}{s}-1\right)+\frac{s}{2 d} \mathbb{P}_{a}\left(\frac{\bar{\varphi}_{\sigma}(X)}{\varphi_{\sigma}(X)} \leq \frac{d}{s}-1\right) \\
& +\frac{s}{2 d} \mathbb{P}_{-a}\left(\frac{\bar{\varphi}_{\sigma}(X)}{\varphi_{\sigma}(X)} \leq \frac{d}{s}-1\right) \\
= & \left(1-\frac{s}{d}\right) \mathbf{P}\left(e^{-\frac{a^{2}}{2 \sigma^{2}}} \cosh \left(\frac{a \xi}{\sigma}\right)>\frac{d}{s}-1\right) \\
& +\frac{s}{2 d} \mathbb{P}_{a}\left(\frac{\bar{\varphi}_{\sigma}(X)}{\varphi_{\sigma}(X)} \leq \frac{d}{s}-1\right)+\frac{s}{2 d} \mathbb{P}_{-a}\left(\frac{\bar{\varphi}_{\sigma}(X)}{\varphi_{\sigma}(X)} \leq \frac{d}{s}-1\right),
\end{aligned}
$$

where $\mathbb{P}_{u}$ denotes the probability distribution of $X$ with density $\varphi_{\sigma}(\cdot-u)$. Note that, for all $x \in \mathbb{R}$,

$$
\frac{\bar{\varphi}_{\sigma}(x)}{\varphi_{\sigma}(x)}=e^{-\frac{a^{2}}{2 \sigma^{2}}} \cosh \left(\frac{a x}{\sigma^{2}}\right) .
$$

Using this formula with $x=\sigma \xi+a$ and $x=\sigma \xi-a$, and the facts that $\cosh (\cdot)$ is an even function and $\xi$ coincides with $-\xi$ in distribution, we obtain

$$
\begin{aligned}
\mathbb{P}_{a}\left(\frac{\bar{\varphi}_{\sigma}(X)}{\varphi_{\sigma}(X)} \leq \frac{d}{s}-1\right) & =\mathbb{P}_{-a}\left(\frac{\bar{\varphi}_{\sigma}(X)}{\varphi_{\sigma}(X)} \leq \frac{d}{s}-1\right) \\
& =\mathbf{P}\left(e^{-\frac{a^{2}}{2 \sigma^{2}}} \cosh \left(\frac{a \xi}{\sigma}+\frac{a^{2}}{\sigma^{2}}\right) \leq \frac{d}{s}-1\right) .
\end{aligned}
$$

Thus, $\tilde{L}=(s / d) \bar{\Psi}(d, s, a)$. Combining this equality with (49) and (54) proves the theorem.

Proof OF THEOREM 2.6. The upper bounds (14), (15) and (16) follow immediately from (2) and Theorems 2.1, 2.4 and 2.3, respectively. We now prove the lower bound (17). To this end, first note that for any $\theta \in \Theta_{d}^{+}(s, a)$ and any $\tilde{\eta} \in \mathcal{T}$ we have

$$
\mathbf{P}_{\theta}\left(S_{\tilde{\eta}} \neq S(\theta)\right)=\mathbf{P}_{\theta}\left(\bigcup_{j=1}^{d}\left\{\tilde{\eta}_{j} \neq \eta_{j}\right\}\right)=1-\prod_{j=1}^{d} p_{j}(\theta),
$$

where $p_{j}(\theta) \triangleq \mathbf{P}_{\theta}\left(\tilde{\eta}_{j}=\eta_{j}\right)$. Hence, for any $\tilde{\eta} \in \mathcal{T}$,

$$
\sup _{\theta \in \Theta_{d}^{+}(s, a)} \mathbf{P}_{\theta}\left(S_{\tilde{\eta}} \neq S(\theta)\right) \geq \max _{\theta \in \Theta^{\prime}} \mathbf{P}_{\theta}\left(S_{\tilde{\eta}} \neq S(\theta)\right)=1-p_{*},
$$


where $\Theta^{\prime}$ is the subset of $\Theta_{d}^{+}(s, a)$ defined in the proof of Theorem 2.2, and $p_{*}=$ $\min _{\theta \in \Theta^{\prime}} \prod_{j=1}^{d} p_{j}(\theta)$. fore,

Next, for any selector $\tilde{\eta}$ we have $\mathbf{P}_{\theta}\left(S_{\tilde{\eta}} \neq S(\theta)\right) \geq \mathbf{P}_{\theta}(|\tilde{\eta}-\eta|=1)$. There-

$$
\sup _{\theta \in \Theta_{d}^{+}(s, a)} \mathbf{P}_{\theta}\left(S_{\tilde{\eta}} \neq S(\theta)\right) \geq \frac{1}{\left|\Theta^{\prime}\right|} \sum_{\theta \in \Theta^{\prime}} \mathbf{P}_{\theta}(|\tilde{\eta}-\eta|=1) .
$$

Here, $\mathbf{P}_{\theta}(|\tilde{\eta}-\eta|=1)=\mathbf{P}_{\theta}\left(\bigcup_{j=1}^{d} B_{j}\right)$ with the random events $B_{j}=\left\{\left|\tilde{\eta}_{j}-\eta_{j}\right|=\right.$ 1 , and $\left.\tilde{\eta}_{i}=\eta_{i}, \forall i \neq j\right\}$. Since the events $B_{j}$ are disjoint, for any $\tilde{\eta} \in \mathcal{T}$ we get

$$
\begin{aligned}
\frac{1}{\left|\Theta^{\prime}\right|} \sum_{\theta \in \Theta^{\prime}} \mathbf{P}_{\theta}(|\tilde{\eta}-\eta|=1) \\
=\frac{1}{\left|\Theta^{\prime}\right|} \sum_{\theta \in \Theta^{\prime}} \sum_{j=1}^{d} \mathbf{P}_{\theta}\left(B_{j}\right) \\
=\frac{1}{\left|\Theta^{\prime}\right|} \sum_{j=1}^{d}\left(\sum_{\theta \in \Theta^{\prime}: \theta_{j}=0} \mathbf{P}_{j, 0}\left(\tilde{\eta}_{j}=1\right) \prod_{i \neq j} p_{i}(\theta)\right. \\
\left.\quad+\sum_{\theta \in \Theta^{\prime}: \theta_{j}=a} \mathbf{P}_{j, a}\left(\tilde{\eta}_{j}=0\right) \prod_{i \neq j} p_{i}(\theta)\right) \\
\geq \frac{p_{*}}{\left|\Theta^{\prime}\right|} \sum_{j=1}^{d}\left(\sum_{\theta \in \Theta^{\prime}: \theta_{j}=0} \mathbf{P}_{j, 0}\left(\tilde{\eta}_{j}=1\right)+\sum_{\theta \in \Theta^{\prime}: \theta_{j}=a} \mathbf{P}_{j, a}\left(\tilde{\eta}_{j}=0\right)\right) \\
=\frac{p_{*}}{\left|\Theta^{\prime}\right|} \sum_{j=1}^{d}\left(\sum_{\theta \in \Theta^{\prime}: \theta_{j}=0} \mathbf{E}_{j, 0}\left(\tilde{\eta}_{j}\right)+\sum_{\theta \in \Theta^{\prime}: \theta_{j}=a} \mathbf{E}_{j, a}\left(1-\tilde{\eta}_{j}\right)\right),
\end{aligned}
$$

where $\mathbf{P}_{j, u}$ denotes the distribution of $X_{j}$ when $\theta_{j}=u$. We now bound the righthand side of (58) by following the argument from the last three lines of (50) to the end of the proof of Theorem 2.2. Applying this argument yields that, for any $\tilde{\eta} \in \mathcal{T}$,

$$
\frac{1}{\left|\Theta^{\prime}\right|} \sum_{\theta \in \Theta^{\prime}} \mathbf{P}_{\theta}(|\tilde{\eta}-\eta|=1) \geq p^{*} d \tilde{L} \geq p^{*} s \Psi_{+}(d, s, a) .
$$

Combining (56), (57) and (59), we find that, for any $\tilde{\eta} \in \mathcal{T}$,

$$
\begin{aligned}
\sup _{\theta \in \Theta_{d}^{+}(s, a)} \mathbf{P}_{\theta}\left(S_{\tilde{\eta}} \neq S(\theta)\right) & \geq \min _{0 \leq p^{*} \leq 1} \max \left\{1-p^{*}, p^{*} s \Psi_{+}(d, s, a)\right\} \\
& =\frac{s \Psi_{+}(d, s, a)}{1+s \Psi_{+}(d, s, a)}
\end{aligned}
$$


We now prove the lower bound (18). Let the sets $\Theta^{+}$and $\Theta^{-}$and the constants $p_{j}(\theta)$ be the same as in the proof of Theorem 2.5. Then

$$
\sup _{\theta \in \Theta_{d}(s, a)} \mathbf{P}_{\theta}\left(S_{\tilde{\eta}} \neq S(\theta)\right) \geq \max _{\theta \in \Theta^{+} \cup \Theta^{-}} \mathbf{P}_{\theta}\left(S_{\tilde{\eta}} \neq S(\theta)\right)=1-\bar{p},
$$

where $\bar{p}=\min _{\theta \in \Theta^{+} \cup \Theta^{-}} \prod_{j=1}^{d} p_{j}(\theta)$.

For any selector $\tilde{\eta}$, we use that $\mathbf{P}_{\theta}\left(S_{\tilde{\eta}} \neq S(\theta)\right) \geq \mathbf{P}_{\theta}(|\tilde{\eta}-\eta|=1)$ and, therefore,

$$
\begin{aligned}
\sup _{\theta \in \Theta_{d}(s, a)} \mathbf{P}_{\theta}\left(S_{\tilde{\eta}} \neq S(\theta)\right) \geq & \frac{1}{2\left|\Theta^{+}\right|} \sum_{\theta \in \Theta^{+}} \mathbf{P}_{\theta}(|\tilde{\eta}-\eta|=1) \\
& +\frac{1}{2\left|\Theta^{-}\right|} \sum_{\theta \in \Theta^{-}} \mathbf{P}_{\theta}(|\tilde{\eta}-\eta|=1) .
\end{aligned}
$$

We continue along the same lines as in the proof of (58) to get, for any separable selector $\tilde{\eta}$,

$$
\begin{aligned}
\sup _{\theta \in \Theta_{d}(s, a)} \mathbf{P}_{\theta}\left(S_{\tilde{\eta}} \neq S(\theta)\right) \\
\geq \frac{\bar{p}}{2\left|\Theta^{+}\right|} \sum_{j=1}^{d}\left(\sum_{\theta \in \Theta^{+}: \theta_{j}=0} \mathbf{E}_{j, 0}\left(\tilde{\eta}_{j}\right)+\sum_{\theta \in \Theta^{+}: \theta_{j}=a} \mathbf{E}_{j, a}\left(1-\tilde{\eta}_{j}\right)\right) \\
\quad+\frac{\bar{p}}{2\left|\Theta^{-}\right|} \sum_{j=1}^{d}\left(\sum_{\theta \in \Theta^{-}: \theta_{j}=0} \mathbf{E}_{j, 0}\left(\tilde{\eta}_{j}\right)+\sum_{\theta \in \Theta^{-}: \theta_{j}=-a} \mathbf{E}_{j,-a}\left(1-\tilde{\eta}_{j}\right)\right) \\
\geq \frac{\bar{p}}{2} \sum_{j=1}^{d}\left(\left(1-\frac{s}{d}\right) \mathbf{E}_{j, 0}\left(\tilde{\eta}_{j}\right)+\frac{s}{d} \mathbf{E}_{j, a}\left(1-\tilde{\eta}_{j}\right)\right) \\
\quad+\frac{\bar{p}}{2} \sum_{j=1}^{d}\left(\left(1-\frac{s}{d}\right) \mathbf{E}_{j, 0}\left(\tilde{\eta}_{j}\right)+\frac{s}{d} \mathbf{E}_{j,-a}\left(1-\tilde{\eta}_{j}\right)\right) \\
=\bar{p} \sum_{j=1}^{d}\left(\left(1-\frac{s}{d}\right) \mathbf{E}_{j, 0}\left(\bar{\eta}_{j}\right)+\frac{s}{d} \overline{\mathbf{E}}_{j}\left(1-\tilde{\eta}_{j}\right)\right),
\end{aligned}
$$

where again $\overline{\mathbf{E}}_{j}$ denotes the expected value with respect to $\overline{\mathbf{P}}_{j}=\frac{1}{2}\left(\mathbf{P}_{j, a}+\mathbf{P}_{j,-a}\right)$. Analogously to the proof of Theorem 2.5, the expression in the last display can be further bounded from below by $\bar{p} d \tilde{L}=\bar{p} s \bar{\Psi}(d, s, a)$. Thus,

$$
\begin{aligned}
\sup _{\theta \in \Theta_{d}(s, a)} \mathbf{P}_{\theta}\left(S_{\tilde{\eta}} \neq S(\theta)\right) & \geq \min _{0 \leq \bar{p} \leq 1} \max \{1-\bar{p}, \bar{p} s \bar{\Psi}(d, s, a)\} \\
& =\frac{s \bar{\Psi}(d, s, a)}{1+s \bar{\Psi}(d, s, a)} .
\end{aligned}
$$


Proof of THEOREM 4.2. (i) It follows from the second inequality in (48) that

$$
\sup _{\theta \in \Theta_{d}(s, a)} \frac{1}{s} \mathbf{E}_{\theta}|\hat{\eta}-\eta| \leq 2\left(\frac{d}{s}-1\right) \Phi(-t / \sigma)+2 \Phi\left(-(a-t)_{+} / \sigma\right),
$$

where $t=\frac{a}{2}+\frac{\sigma^{2}}{a} \log \left(\frac{d}{s}-1\right)$ is the threshold (5). Since $a^{2} \geq 2 \sigma^{2} \log (d / s-1)$ we get that $a \geq t$ and that $t>a / 2$, which is equivalent to $t>a-t$. Furthermore, $\left(\frac{d}{s}-1\right) e^{-t^{2} /\left(2 \sigma^{2}\right)}=e^{-(a-t)^{2} /\left(2 \sigma^{2}\right)}$. These remarks and (31) imply that

$$
\begin{aligned}
\left(\frac{d}{s}-1\right) \Phi(-t / \sigma) & \leq \sqrt{\frac{2}{\pi}} \frac{\exp \left(-(a-t)^{2} /\left(2 \sigma^{2}\right)\right)}{(a-t) / \sigma+\sqrt{(a-t)^{2} / \sigma^{2}+8 / \pi}} \\
& \leq \frac{\exp \left(-(a-t)^{2} /\left(2 \sigma^{2}\right)\right)}{(a-t) / \sigma+\sqrt{(a-t)^{2} / \sigma^{2}+4}} \\
& \leq \sqrt{\frac{\pi}{2}} \Phi\left(-\frac{a-t}{\sigma}\right) .
\end{aligned}
$$

Combining this with (60), we get

$$
\sup _{\theta \in \Theta_{d}(s, a)} \frac{1}{s} \mathbf{E}_{\theta}|\hat{\eta}-\eta| \leq(2+\sqrt{2 \pi}) \Phi\left(-\frac{a-t}{\sigma}\right) .
$$

Now, to prove (33) it remains to note that under assumption (32),

$$
\frac{a-t}{\sigma}=\frac{a}{2 \sigma}-\frac{\sigma}{a} \log \left(\frac{d}{s}-1\right)=\frac{a^{2}-2 \sigma^{2} \log ((d-s) / s)}{2 a \sigma} \geq \Delta .
$$

Indeed, assumption (32) states that $a \geq a_{0} \triangleq \sigma(2 \log ((d-s) / s)+W)^{1 / 2}$, and the function $a \mapsto\left(a^{2}-2 \sigma^{2} \log ((d-s) / s)\right) / a$ is monotonically increasing in $a>0$. On the other hand,

$$
\left(a_{0}^{2}-2 \sigma^{2} \log ((d-s) / s)\right) /\left(2 a_{0} \sigma\right)=\Delta .
$$

(ii) We now prove (36). By Theorem 2.2,

$\inf _{\tilde{\eta}} \sup _{\theta \in \Theta_{d}(s, a)} \frac{1}{s} \mathbf{E}_{\theta}|\tilde{\eta}-\eta| \geq \frac{s^{\prime}}{s} \Phi\left(-\frac{a}{2 \sigma}+\frac{\sigma}{a} \log \left(\frac{d}{s}-1\right)\right)-4 \frac{s^{\prime}}{s} \exp \left(-\frac{\left(s-s^{\prime}\right)^{2}}{2 s}\right)$.

Here,

$$
-\frac{a}{2 \sigma}+\frac{\sigma}{a} \log \left(\frac{d}{s}-1\right)=\frac{2 \sigma^{2} \log ((d-s) / s)-a^{2}}{2 \sigma a} .
$$

Observe that the function $a \mapsto\left(2 \sigma^{2} \log ((d-s) / s)-a^{2}\right) / a$ is monotonically decreasing in $a>0$ and that assumption (35) states that $a \leq a_{0}$. In view of (61), the 
value of its minimum for $a \leq a_{0}$ is equal to $-\Delta$. The bound (36) now follows by the monotonicity of $\Phi(\cdot)$.

Proof OF THEOREM 4.3. Assume without loss of generality that $d$ is large enough to have $\left(d-s_{d}\right) / s_{d}>1$. We apply Theorem 4.2 with $W=$ $A \sqrt{2 \log \left(\left(d-s_{d}\right) / s_{d}\right)}$. Then

$$
\Delta^{2}=\frac{A^{2} \sqrt{2 \log \left(\left(d-s_{d}\right) / s_{d}\right)}}{4\left(\sqrt{2 \log \left(\left(d-s_{d}\right) / s_{d}\right)}+A\right)} .
$$

By assumption, there exists $v>0$ such that $(2+v) s_{d} \leq d$ for all $d$ large enough. Equivalently, $d / s_{d}-1 \geq 1+v$ and, therefore, using the monotonicity argument, we find

$$
\Delta^{2} \geq \frac{A^{2} \sqrt{2 \log (1+v)}}{\sqrt{2 \log (1+v)}+A} \rightarrow \infty \quad \text { as } A \rightarrow \infty .
$$

This and (33) imply part (i) of the theorem.

Part (ii) follows from (36) by noticing that $\Delta^{2} \leq \sup _{x>0} \frac{A^{2} x}{4(x+A)}=A^{2} / 4$ for any fixed $A>0$. Now, for $s$ large enough, let us put $s^{\prime}=(1-\varepsilon) s$ for some $\varepsilon$ in $(0,1)$, fixed. Thus, the lower bound of the risk becomes

$$
(1-\varepsilon) \Phi(-\Delta)-4 \exp \left(-\frac{s}{2}(1-\varepsilon)^{2}\right)>0,
$$

for $s$ large enough.

ProOF OF THEOREM 4.4. Throughout the proof, we assume without loss of generality that $d$ is large enough to have $s_{d} \geq 2$, and $\left(d-s_{d}\right) / s_{d}>1$. Set $W_{*}(s) \triangleq$ $4(\log s+\sqrt{\log s \log (d-s)})$, and notice that

$$
\begin{aligned}
\frac{W_{*}\left(s_{d}\right)}{2 \sqrt{2 \log \left(\left(d-s_{d}\right) / s_{d}\right)+W_{*}\left(s_{d}\right)}} & =\sqrt{2 \log s_{d}}, \\
2 \log \left(\left(d-s_{d}\right) / s_{d}\right)+W_{*}\left(s_{d}\right) & =2\left(\sqrt{\log \left(d-s_{d}\right)}+\sqrt{\log s_{d}}\right)^{2} .
\end{aligned}
$$

If (40) holds, we have $W_{d} \geq W_{*}\left(s_{d}\right)$ for all $d$ large enough. By the monotonicity of the quantity $\Delta$ defined in (34) with respect to $W$, this implies

$$
\begin{aligned}
\Delta_{d} & \triangleq \frac{W_{d}}{2 \sqrt{2 \log \left(\left(d-s_{d}\right) / s_{d}\right)+W_{d}}} \\
& \geq \frac{W_{*}\left(s_{d}\right)}{2 \sqrt{2 \log \left(\left(d-s_{d}\right) / s_{d}\right)+W_{*}\left(s_{d}\right)}}=\sqrt{2 \log s_{d}} .
\end{aligned}
$$


Now, by Theorem 4.2 and using (31) we may write

$$
\begin{aligned}
\sup _{\theta \in \Theta_{d}\left(s_{d}, a_{d}\right)} \mathbf{E}_{\theta}|\hat{\eta}-\eta| & \leq(2+\sqrt{2 \pi}) s_{d} \Phi\left(-\Delta_{d}\right) \\
& \leq 3 s_{d} \min \left\{1, \frac{1}{\Delta_{d}}\right\} \exp \left(-\frac{\Delta_{d}^{2}}{2}\right) \\
& =3 \min \left\{1, \frac{1}{\Delta_{d}}\right\} \exp \left(-\frac{\Delta_{d}^{2}-2 \log s_{d}}{2}\right) .
\end{aligned}
$$

This and (64) imply that, for all $d$ large enough,

$$
\sup _{\theta \in \Theta_{d}\left(s_{d}, a_{d}\right)} \mathbf{E}_{\theta}|\hat{\eta}-\eta| \leq 3 \min \left\{1, \frac{1}{\sqrt{2 \log s_{d}}}\right\} \text {. }
$$

Since $s_{d} \rightarrow \infty$, part (i) of the theorem follows.

We now prove part (ii) of the theorem. It suffices to consider $W_{d}>0$ for all $d$ large enough since for nonpositive $W_{d}$ almost full recovery is impossible and the result follows from part (ii) of Theorem 4.3. If (42) holds, there exists $A<1$ such that $W_{d} \leq A W_{*}\left(s_{d}\right)$ for all $d$ large enough. By the monotonicity of the quantity $\Delta$ defined in (34) with respect to $W$ and in view of equation (62), this implies

$$
\begin{aligned}
\Delta_{d}^{2}- & 2 \log s_{d} \\
\leq & \frac{A^{2} W_{*}^{2}\left(s_{d}\right)}{4\left(2 \log \left(\left(d-s_{d}\right) / s_{d}\right)+A W_{*}\left(s_{d}\right)\right)} \\
& -\frac{W_{*}^{2}\left(s_{d}\right)}{4\left(2 \log \left(\left(d-s_{d}\right) / s_{d}\right)+W_{*}\left(s_{d}\right)\right)} \\
= & \frac{(A-1) W_{*}^{2}\left(s_{d}\right)\left(A W_{*}\left(s_{d}\right)+2(A+1) \log \left(\left(d-s_{d}\right) / s_{d}\right)\right)}{4\left(2 \log \left(\left(d-s_{d}\right) / s_{d}\right)+A W_{*}\left(s_{d}\right)\right)\left(2 \log \left(\left(d-s_{d}\right) / s_{d}\right)+W_{*}\left(s_{d}\right)\right)} \\
\leq & \frac{(A-1) A W_{*}^{2}\left(s_{d}\right)}{4\left(2 \log \left(\left(d-s_{d}\right) / s_{d}\right)+W_{*}\left(s_{d}\right)\right)} \\
= & \frac{2(A-1) A\left(\log s_{d}+\sqrt{\log s_{d} \log \left(d-s_{d}\right)}\right)^{2}}{\left(\sqrt{\log \left(d-s_{d}\right)}+\sqrt{\log s_{d}}\right)^{2}}=2(A-1) A \log s_{d},
\end{aligned}
$$

where we have used the fact that $A<1$ and equations (62), (63). Next, by Theorem 4.2 and using (31), we have for $s^{\prime}=s_{d} / 2$,

$$
\inf _{\tilde{\eta}} \sup _{\theta \in \Theta_{d}\left(s_{d}, a_{d}\right)} \mathbf{E}_{\theta}|\tilde{\eta}-\eta| \geq \frac{s_{d}}{2}\left(\Phi\left(-\Delta_{d}\right)-4 \exp \left(-\frac{s_{d}}{8}\right)\right)
$$

and

$$
\frac{s_{d}}{2} \Phi\left(-\Delta_{d}\right) \geq \frac{s_{d}}{8} \min \left\{\frac{1}{2}, \frac{1}{\Delta_{d}}\right\} \exp \left(-\frac{\Delta_{d}^{2}}{2}\right)
$$




$$
=\frac{1}{8} \min \left\{\frac{1}{2}, \frac{1}{\Delta_{d}}\right\} \exp \left(-\frac{\Delta_{d}^{2}-2 \log s_{d}}{2}\right) .
$$

Combining this inequality with (66), we find that, for all $d$ large enough,

$$
\inf _{\tilde{\eta}} \sup _{\theta \in \Theta_{d}\left(s_{d}, a_{d}\right)} \mathbf{E}_{\theta}|\tilde{\eta}-\eta| \geq \frac{1}{8} \min \left\{\frac{1}{2}, \frac{1}{\Delta_{d}}\right\} e^{(1-A) A \log s_{d}}-2 s_{d} e^{-s_{d} / 8} .
$$

Since $A<1$ and $\Delta_{d} \leq A \sqrt{2 \log s_{d}}$ by (66), the last expression tends to $\infty$ as $s_{d} \rightarrow \infty$. This proves part (ii) of the theorem.

Proof of Theorem 5.1. By (48), for any $\theta \in \Theta_{d}\left(s_{d}, a_{d}\right)$, and any $t>0$ we have

$$
\mathbf{E}_{\theta}|\hat{\eta}-\eta| \leq d \mathbf{P}(|\xi| \geq t / \sigma)+s_{d} \mathbf{P}\left(|\xi|>\left(a_{d}-t\right)_{+} / \sigma\right),
$$

where $\xi$ is a standard normal random variable. It follows that, for any $a_{d} \geq a_{d}^{*}$, any $\theta \in \Theta_{d}\left(s_{d}, a_{d}\right)$, and any $t>0$,

$$
\mathbf{E}_{\theta}|\hat{\eta}-\eta| \leq d \mathbf{P}(|\xi| \geq t / \sigma)+s_{d} \mathbf{P}\left(|\xi|>\left(a_{d}^{*}-t\right)_{+} / \sigma\right)
$$

Without loss of generality assume that $d \geq 6$ and $2 \leq s_{d} \leq d / 2$. Then, using the inequality $\sqrt{x}-\sqrt{y} \leq(x-y) / \sqrt{2 y}, \forall x>y>0$, we find that, for $t=\sigma \sqrt{2 \log d}$,

$$
\begin{aligned}
\left(a_{d}^{*}-t\right)_{+} / \sigma & \geq \sqrt{2}\left(\sqrt{\log \left(d-s_{d}\right)}-\sqrt{\log d}+\sqrt{\log \left(s_{d}\right)}\right) \\
& \geq \sqrt{2 \log \left(s_{d}\right)}-\log \left(\frac{d}{d-s_{d}}\right) / \sqrt{\log \left(d-s_{d}\right)} \\
& \geq \sqrt{2 \log \left(s_{d}\right)}-(\log 2) / \sqrt{\log (d / 2)}>0 .
\end{aligned}
$$

From this we also easily deduce that, for $2 \leq s_{d} \leq d / 2$, we have $\left(\left(a_{d}^{*}-\right.\right.$ $\left.t)_{+} / \sigma\right)^{2} / 2 \geq \log \left(s_{d}\right)-\sqrt{2} \log 2$. Combining these remarks with (31) and (43), we find

$$
\sup _{\theta \in \Theta_{d}\left(s_{d}, a_{d}\right)} \mathbf{E}_{\theta}|\hat{\eta}-\eta| \leq \frac{1}{\sqrt{2 \log d}}+\frac{s_{d} \exp \left(-\log \left(s_{d}\right)+\sqrt{2} \log 2\right)}{\sqrt{2 \log \left(s_{d}\right)}},
$$

which immediately implies the theorem by taking the limit as $d \rightarrow \infty$.

PROOF OF THEOREM 5.2. Throughout the proof, we will write for brevity $s_{d}=s, a_{d}=a, A_{d}=A$, and set $\sigma=1$. Since $\Theta_{d}(s, a) \subseteq \Theta_{d}\left(s, a_{0}(s, A)\right)$ for all $a \geq a_{0}(s, A)$, it suffices to prove that

$$
\lim _{d \rightarrow \infty} \sup _{\theta \in \Theta_{d}\left(s, a_{0}(s, A)\right)} \frac{1}{s} \mathbf{E}_{\theta}\left|\hat{\eta}^{\mathrm{ad}}-\eta\right|=0 .
$$

Here, $s \leq s_{d}^{*}$ and recall that throughout this section we assume that $s_{d}^{*} \leq d / 4$; since we deal with asymptotics as $d / s_{d}^{*} \rightarrow \infty$, the latter assumption is without loss of generality in the current proof. 
If $s<g_{M}$, let $m_{0} \in\{2, \ldots, M\}$ be the index such that $g_{m_{0}}$ is the minimal element of the grid, which is greater than the true underlying $s$. Thus, $g_{m_{0}} / 2=$ $g_{m_{0}-1} \leq s<g_{m_{0}}$. If $s \in\left[g_{M}, s_{d}^{*}\right]$, we set $m_{0}=M$. In both cases,

$$
s \geq g_{m_{0}} / 2 .
$$

We decompose the risk as follows:

$$
\frac{1}{s} \mathbf{E}_{\theta}\left|\hat{\eta}^{\mathrm{ad}}-\eta\right|=I_{1}+I_{2},
$$

where

$$
\begin{aligned}
& I_{1}=\frac{1}{s} \mathbf{E}_{\theta}\left(\left|\hat{\eta}\left(g_{\hat{m}}\right)-\eta\right| I\left(\hat{m} \leq m_{0}\right)\right), \\
& I_{2}=\frac{1}{s} \mathbf{E}_{\theta}\left(\left|\hat{\eta}\left(g_{\hat{m}}\right)-\eta\right| I\left(\hat{m} \geq m_{0}+1\right)\right) .
\end{aligned}
$$

We now evaluate $I_{1}$. Using the fact that $\hat{\eta}_{j}\left(g_{m}\right)$ is monotonically increasing in $m$ and the definition of $\hat{m}$, we obtain that, on the event $\left\{\hat{m} \leq m_{0}\right\}$,

$$
\begin{aligned}
\left|\hat{\eta}\left(g_{\hat{m}}\right)-\hat{\eta}\left(g_{m_{0}}\right)\right| & \leq \sum_{m=\hat{m}+1}^{m_{0}}\left|\hat{\eta}\left(g_{m}\right)-\hat{\eta}\left(g_{m-1}\right)\right| \\
& =\sum_{m=\hat{m}+1}^{m_{0}} \sum_{j=1}^{d}\left(\hat{\eta}_{j}\left(g_{m}\right)-\hat{\eta}_{j}\left(g_{m-1}\right)\right) \\
& =\sum_{m=\hat{m}+1}^{m_{0}} \sum_{j=1}^{d} I\left(w\left(g_{m}\right) \leq\left|X_{j}\right|<w\left(g_{m-1}\right)\right) \\
& \leq \tau \sum_{m=\hat{m}+1}^{m_{0}} g_{m} \leq \tau s \sum_{m=2}^{m_{0}} 2^{m-m_{0}+1} \leq 4 \tau s,
\end{aligned}
$$

where we have used the equality $g_{m}=2^{m}$ and (68). Thus,

$$
\begin{aligned}
I_{1} & \leq \frac{1}{s} \mathbf{E}_{\theta}\left(\left|\hat{\eta}\left(g_{\hat{m}}\right)-\hat{\eta}\left(g_{m_{0}}\right)\right| I\left(\hat{m} \leq m_{0}\right)\right)+\frac{1}{s} \mathbf{E}_{\theta}\left|\hat{\eta}\left(g_{m_{0}}\right)-\eta\right| \\
& \leq 4 \tau+\frac{1}{s} \mathbf{E}_{\theta}\left|\hat{\eta}\left(g_{m_{0}}\right)-\eta\right| .
\end{aligned}
$$

Next, note that the first inequality in (48) is true for any $t>0$. Applying it with $t=w\left(g_{m_{0}}\right)$, we obtain

$$
\begin{aligned}
\frac{1}{s} \mathbf{E}_{\theta}\left|\hat{\eta}\left(g_{m_{0}}\right)-\eta\right| \leq & \frac{d}{s} \mathbf{P}\left(|\xi| \geq w\left(g_{m_{0}}\right)\right) \\
& +\mathbf{P}\left(|\xi|>\left(a_{0}(s, A)-w\left(g_{m_{0}}\right)\right)_{+}\right)
\end{aligned}
$$


where $\xi$ is a standard Gaussian random variable. Using the bound on the Gaussian tail probability and the fact that $g_{m_{0}}>s \geq g_{m_{0}} / 2$, we get

$$
\begin{aligned}
\frac{d}{s} \mathbf{P}\left(|\xi| \geq w\left(g_{m_{0}}\right)\right) & \leq \frac{d / s}{d / g_{m_{0}}-1} \frac{\pi^{-1 / 2}}{\sqrt{\log \left(d / g_{m_{0}}-1\right)}} \\
& \leq \frac{d}{d-2 s} \frac{2 \pi^{-1 / 2}}{\sqrt{\log (d / s-1)}} \leq \frac{4 \pi^{-1 / 2}}{\sqrt{\log \left(d / s_{d}^{*}-1\right)}}
\end{aligned}
$$

To bound the second probability on the right-hand side of (70), we use the following lemma.

LEMMA A.1. Under the assumptions of Theorem 5.2, for any $m \geq m_{0}$ we have

$$
\mathbf{P}\left(|\xi|>\left(a_{0}(s, A)-w\left(g_{m}\right)\right)_{+}\right) \leq\left(\log \left(d / s_{d}^{*}-1\right)\right)^{-\frac{1}{2}}
$$

Combining (70), (71) and (72) with $m=m_{0}$, we find

$$
\frac{1}{s} \mathbf{E}_{\theta}\left|\hat{\eta}\left(g_{m_{0}}\right)-\eta\right| \leq \frac{4 \pi^{-1 / 2}+1}{\sqrt{\log \left(d / s_{d}^{*}-1\right)}}
$$

which together with (69) leads to the bound

$$
I_{1} \leq 4 \tau+\frac{4 \pi^{-1 / 2}+1}{\sqrt{\log \left(d / s_{d}^{*}-1\right)}} .
$$

We now turn to the evaluation of $I_{2}$. It is enough to consider the case $m_{0} \leq M-1$ since $I_{2}=0$ when $m_{0}=M$. We have

$$
\begin{aligned}
I_{2} & =\frac{1}{s} \sum_{m=m_{0}+1}^{M} \mathbf{E}_{\theta}\left(\left|\hat{\eta}\left(g_{\hat{m}}\right)-\eta\right| I(\hat{m}=m)\right) \\
& \leq \frac{1}{s} \sum_{m=m_{0}+1}^{M}\left(\mathbf{E}_{\theta}\left|\hat{\eta}\left(g_{m}\right)-\eta\right|^{2}\right)^{1 / 2}\left(\mathbf{P}_{\theta}(\hat{m}=m)\right)^{1 / 2} .
\end{aligned}
$$

By definition, the event $\{\hat{m}=m\}$ occurs implies that $\sum_{j=1}^{d} I\left(w_{m} \leq\left|X_{j}\right|<\right.$ $\left.w_{m-1}\right)>\tau g_{m} \triangleq v_{m}$, where we set for brevity $w_{m}=w\left(g_{m}\right)$. Thus,

$$
\mathbf{P}_{\theta}(\hat{m}=m) \leq \mathbf{P}_{\theta}\left(\sum_{j=1}^{d} I\left(w_{m} \leq\left|X_{j}\right|<w_{m-1}\right)>v_{m}\right) .
$$


By Bernstein's inequality, for any $t>0$ we have

$$
\begin{gathered}
\mathbf{P}_{\theta}\left(\sum_{j=1}^{d} I\left(w_{m} \leq\left|X_{j}\right|<w_{m-1}\right)-\mathbf{E}_{\theta}\left(\sum_{j=1}^{d} I\left(w_{m} \leq\left|X_{j}\right|<w_{m-1}\right)\right)>t\right) \\
\quad \leq \exp \left(-\frac{t^{2} / 2}{\sum_{j=1}^{d} \mathbf{E}_{\theta}\left(I\left(w_{m} \leq\left|X_{j}\right|<w_{m-1}\right)\right)+2 t / 3}\right),
\end{gathered}
$$

where we have used that, for random variables with values in $\{0,1\}$, the variance is smaller than the expectation.

Now, similar to (48), for any $\theta \in \Theta_{d}\left(s, a_{0}(s, A)\right)$,

$$
\begin{aligned}
& \mathbf{E}_{\theta}\left(\sum_{j=1}^{d} I\left(w_{m} \leq\left|X_{j}\right|<w_{m-1}\right)\right) \\
& \quad \leq d \mathbf{P}\left(w_{m} \leq|\xi|<w_{m-1}\right)+\sum_{j: \theta_{j} \neq 0} \mathbf{P}\left(\left|\theta_{j}+\xi\right|<w_{m-1}\right) \\
& \quad \leq d \mathbf{P}\left(|\xi| \geq w_{m}\right)+s \mathbf{P}\left(|\xi|>-\left(a_{0}(s, A)-w_{m-1}\right)_{+}\right),
\end{aligned}
$$

where $\xi$ is a standard Gaussian random variable. Since $m \geq m_{0}+1$, from Lemma A.1 we get

$$
\mathbf{P}\left(|\xi|>\left(a_{0}(s, A)-w_{m-1}\right)_{+}\right) \leq\left(\log \left(d / s_{d}^{*}-1\right)\right)^{-\frac{1}{2}} .
$$

Next, using the bound on the Gaussian tail probability and the inequalities $g_{m} \leq$ $s_{d}^{*} \leq d / 4$, we find

$$
d \mathbf{P}\left(|\xi| \geq w_{m}\right) \leq \frac{d}{d / g_{m}-1} \frac{\pi^{-1 / 2}}{\sqrt{\log \left(d / g_{m}-1\right)}} \leq \frac{(4 / 3) \pi^{-1 / 2} g_{m}}{\sqrt{\log \left(d / s_{d}^{*}-1\right)}} .
$$

We now deduce from (78) and (79), and the inequality $s \leq g_{m}$ for $m \geq m_{0}+1$, that

$$
\mathbf{E}_{\theta}\left(\sum_{j=1}^{d} I\left(w_{m} \leq\left|X_{j}\right|<w_{m-1}\right)\right) \leq \frac{\left((4 / 3) \pi^{-1 / 2}+1\right) g_{m}}{\sqrt{\log \left(d / s_{d}^{*}-1\right)}} \leq 2 \tau g_{m} .
$$

Taking in (77) $t=3 \tau g_{m}=3 v_{m}$ and using (80), we find

$$
\mathbf{P}_{\theta}\left(\sum_{j=1}^{d} I\left(w_{m} \leq\left|X_{j}\right|<w_{m-1}\right)>v_{m}\right) \leq \exp \left(-C_{1} v_{m}\right)=\exp \left(-C_{1} 2^{m} \tau\right)
$$

for some absolute constant $C_{1}>0$. This implies

$$
\mathbf{P}_{\theta}(\hat{m}=m) \leq \exp \left(-C_{1} 2^{m} \tau\right) \text {. }
$$


On the other hand, notice that the bounds (70), and (71) are valid not only for $g_{m_{0}}$ but also for any $g_{m}$ with $m \geq m_{0}+1$. Using this observation and Lemma A.1 we get that, for any $\theta \in \Theta_{d}\left(s, a_{0}(s, A)\right)$ and any $m \geq m_{0}+1$,

$$
\begin{aligned}
\mathbf{E}_{\theta}\left|\hat{\eta}\left(g_{m}\right)-\eta\right| & \leq s\left[\frac{d / s}{d / g_{m}-1} \frac{\pi^{-1 / 2}}{\sqrt{\log \left(d / g_{m}-1\right)}}+\left(\log \left(d / s_{d}^{*}-1\right)\right)^{-\frac{1}{2}}\right] \\
& \leq \frac{\left((4 / 3) \pi^{-1 / 2}+1\right) g_{m}}{\sqrt{\log \left(d / s_{d}^{*}-1\right)}} \triangleq \tau^{\prime} g_{m}=\tau^{\prime} 2^{m},
\end{aligned}
$$

where the last inequality follows from the same argument as in (79). We denote by $\operatorname{Var}_{\theta}\left(\left|\hat{\eta}\left(g_{m}\right)-\eta\right|\right)$ the variance of $\left|\hat{\eta}\left(g_{m}\right)-\eta\right|$. Observing that $\left|\hat{\eta}\left(g_{m}\right)-\eta\right|$ is a sum of independent Bernoulli random variables, we get

$$
\begin{aligned}
\mathbf{E}_{\theta}\left|\hat{\eta}\left(g_{m}\right)-\eta\right|^{2} & =\operatorname{Var}_{\theta}\left(\left|\hat{\eta}\left(g_{m}\right)-\eta\right|\right)+\left(\mathbf{E}_{\theta}\left|\hat{\eta}\left(g_{m}\right)-\eta\right|\right)^{2} \\
& \leq \mathbf{E}_{\theta}\left|\hat{\eta}\left(g_{m}\right)-\eta\right|+\left(\mathbf{E}_{\theta}\left|\hat{\eta}\left(g_{m}\right)-\eta\right|\right)^{2}
\end{aligned}
$$

Using (82) and the fact that $\tau^{\prime}$ is bounded, we get that

$$
\mathbf{E}_{\theta}\left|\hat{\eta}\left(g_{m}\right)-\eta\right|^{2} \leq C_{2} \tau^{\prime} 2^{2 m}
$$

for some absolute constant $C_{2}>0$.

Now, we plug (81) and (83) in (75) to obtain

$$
\begin{aligned}
I_{2} & \leq \frac{\left(C_{2} \tau^{\prime}\right)^{1 / 2}}{s} \sum_{m=m_{0}+1}^{M} 2^{m} \exp \left(-C_{1} 2^{m-1} \tau\right) \\
& \leq C_{3}\left(\tau^{\prime}\right)^{1 / 2} \tau^{-1} \exp \left(-C_{1} 2^{m_{0}-1} \tau\right) \leq C_{3}\left(\tau^{\prime}\right)^{1 / 2} \tau^{-1}
\end{aligned}
$$

for some absolute constant $C_{3}>0$. Notice that $\left(\tau^{\prime}\right)^{1 / 2}=O\left(\left(\log \left(d / s_{d}^{*}-1\right)\right)^{-\frac{1}{4}}\right)$ as $d / s_{d}^{*} \rightarrow \infty$ while $\tau^{-1}=O\left(\left(\log \left(d / s_{d}^{*}-1\right)\right)^{\frac{1}{7}}\right)$. Thus, $I_{2}=o(1)$ as $d \rightarrow \infty$. Since from (74) we also get that $I_{1}=o(1)$ as $d \rightarrow \infty$, the proof is complete.

Proof of Lemma A.1. Let first $s<g_{M}$. Then, by definition of $m_{0}$, we have $s<g_{m_{0}}$. Therefore, $s<g_{m}$ for $m \geq m_{0}$, and we have $w\left(g_{m}\right)<w(s)$. It follows that

$$
a_{0}(s, A)-w\left(g_{m}\right) \geq a_{0}(s, A)-w(s) \geq \frac{\sqrt{A}}{2 \sqrt{2}} \min \left(\frac{\sqrt{A}}{\sqrt{2}}, \log ^{1 / 4}(d / s-1)\right),
$$

where we have used the elementary inequalities

$$
\sqrt{x+y}-\sqrt{x} \geq y /(2 \sqrt{x+y}) \geq(2 \sqrt{2})^{-1} \min (y / \sqrt{x}, \sqrt{y})
$$


with $x=2 \log (d / s-1)$ and $y=A \sqrt{\log (d / s-1)}$. By assumption, $A \geq$ $16 \sqrt{\log \log \left(d / s_{d}^{*}-1\right)}$, so that we get

$$
a_{0}(s, A)-w\left(g_{m}\right) \geq a_{0}(s, A)-w(s) \geq 4\left(\log \log \left(\frac{d}{s_{d}^{*}}-1\right)\right)^{1 / 2} .
$$

This and the standard bound on the Gaussian tail probability imply

$$
\begin{aligned}
\mathbf{P}\left(|\xi|>\left(a_{0}(s, A)-w\left(g_{m}\right)\right)_{+}\right) & \leq \exp \left(-\left(a_{0}(s, A)-w\left(g_{m}\right)\right)^{2} / 2\right) \\
& \leq\left(\log \left(d / s_{d}^{*}-1\right)\right)^{-\frac{1}{2}} .
\end{aligned}
$$

Let now $s \in\left[g_{M}, s_{d}^{*}\right]$. Then $m_{0}=M$ and we need to prove the result only for $m=M$. By definition of $M$, we have $s_{d}^{*} \leq 2 g_{M}$. This and (84) imply

$$
\begin{aligned}
a_{0}(s, A)-w\left(g_{M}\right) & \geq a_{0}(s, A)-w(s)-\left(w\left(s_{d}^{*} / 2\right)-w\left(s_{d}^{*}\right)\right) \\
& \geq 4\left(\log \log \left(\frac{d}{s_{d}^{*}}-1\right)\right)^{1 / 2}-\left(w\left(s_{d}^{*} / 2\right)-w\left(s_{d}^{*}\right)\right) .
\end{aligned}
$$

Now, using the elementary inequality $\sqrt{\log (x+y)}-\sqrt{\log (x)} \leq y /(2 x \sqrt{\log (x)})$ with $x=d / s_{d}^{*}-1$ and $y=d / s_{d}^{*}$, and the fact that $s_{d}^{*} \leq d / 4$ we find

$$
\begin{aligned}
w\left(s_{d}^{*} / 2\right)-w\left(s_{d}^{*}\right) & \leq \frac{1}{\sqrt{2 \log \left(d / s_{d}^{*}-1\right)}} \frac{d}{d-s_{d}^{*}} \leq \frac{2 \sqrt{2}}{3 \sqrt{\log \left(d / s_{d}^{*}-1\right)}} \\
& \leq 3\left(\log \log \left(\frac{d}{s_{d}^{*}}-1\right)\right)^{1 / 2} .
\end{aligned}
$$

The last two displays yield $a_{0}(s, A)-w\left(g_{M}\right) \geq\left(\log \log \left(\frac{d}{s_{d}^{*}}-1\right)\right)^{1 / 2}$, and we conclude as in (85).

Acknowledgments. We would like to thank Felix Abramovich for helpful discussion of the results.

\section{SUPPLEMENTARY MATERIAL}

Supplement to "Variable selection with Hamming loss" (DOI: 10.1214/17AOS1572SUPP; .pdf). We derive a general lower bound for the minimax risk over all selectors on the class of at most $s$-sparse vectors. The main term of this bound is a Bayes risk with arbitrary prior and the non-asymptotic remainder term is given explicitly. Using this, we prove the lower bounds of Theorems 2.2, 3.2 and 3.3. 


\section{REFERENCES}

[1] Abramovich, F. and Benjamini, Y. (1995). Thresholding of wavelet coefficients as multiple hypotheses testing procedure. In Wavelets and Statistics, Lecture Notes in Statistics 103 5-14. Springer, New York.

[2] Abramovich, F., Benjamini, Y., Donoho, D. L. and Johnstone, I. M. (2006). Adapting to unknown sparsity by controlling the false discovery rate. Ann. Statist. 34 584-653. MR2281879

[3] Abramowitz, M. and Stegun, I. A. (1964). Handbook of Mathematical Functions with Formulas, Graphs, and Mathematical Tables. National Bureau of Standards Applied Mathematics Series 55. For sale by the Superintendent of Documents, U.S. Government Printing Office, Washington, D.C. MR0167642

[4] Arias-Castro, E. and Chen, S. (2017). Distribution-free multiple testing. Electron. J. Stat. 11 1983-2001. MR3651021

[5] Bertin, K. and LecuÉ, G. (2008). Selection of variables and dimension reduction in highdimensional non-parametric regression. Electron. J. Stat. 2 1224-1241. MR2461900

[6] Bogdan, M., van den Berg, E., Sabatti, C., Su, W. and Candès, E. J. (2015). SLOPE-Adaptive variable selection via convex optimization. Ann. Appl. Stat. $91103-$ 1140. MR3418717

[7] Butucea, C., Ingster, Y. I. and Suslina, I. A. (2015). Sharp variable selection of a sparse submatrix in a high-dimensional noisy matrix. ESAIM Probab. Stat. 19 115-134. MR3374872

[8] Butucea, C., Ndaoud, M., Stepanova, N. A. and Tsybakov, A. B. (2018). Supplement to "Variable selection with Hamming loss." DOI:10.1214/17-AOS1572SUPP.

[9] Butucea, C. and Stepanova, N. (2017). Adaptive variable selection in nonparametric sparse additive models. Electron. J. Stat. 11 2321-2357. MR3656494

[10] Collier, O., Comminges, L., Tsybakov, A. B. and Verzelen, N. (2016). Optimal adaptive estimation of linear functionals under sparsity. http://arxiv.org/abs/1611.09744.

[11] Comminges, L. and Dalalyan, A. S. (2012). Tight conditions for consistency of variable selection in the context of high dimensionality. Ann. Statist. 40 2667-2696. MR3097616

[12] GaO, C., LU, Y. and ZhOU, D. (2016). Exact exponent in optimal rates for crowdsourcing. http://arxiv.org/abs/1605.07696.

[13] Genovese, C. R., Jin, J., Wasserman, L. and YaO, Z. (2012). A comparison of the lasso and marginal regression. J. Mach. Learn. Res. 13 2107-2143. MR2956354

[14] Hall, P. and Jin, J. (2010). Innovated higher criticism for detecting sparse signals in correlated noise. Ann. Statist. 38 1686-1732. MR2662357

[15] Ingster, Y. I. and Stepanova, N. A. (2014). Adaptive variable selection in nonparametric sparse regression. J. Math. Sci. 199 184-201.

[16] JI, P. and JIN, J. (2012). UPS delivers optimal phase diagram in high-dimensional variable selection. Ann. Statist. 40 73-103. MR3013180

[17] JIN, J., ZHANG, C.-H. and ZHANG, Q. (2014). Optimality of graphlet screening in high dimensional variable selection. J. Mach. Learn. Res. 15 2723-2772. MR3270749

[18] LAFFERTY, J. and WASSERMAN, L. (2008). Rodeo: Sparse, greedy nonparametric regression. Ann. Statist. 36 28-63. MR2387963

[19] Lehmann, E. L. and Romano, J. P. (2005). Testing Statistical Hypotheses, 3rd ed. Springer, New York. MR2135927

[20] LounicI, K. (2008). Sup-norm convergence rate and sign concentration property of Lasso and Dantzig estimators. Electron. J. Stat. 2 90-102. MR2386087

[21] Meinshausen, N. and BÜHLmann, P. (2006). High-dimensional graphs and variable selection with the lasso. Ann. Statist. 34 1436-1462. MR2278363 
[22] Meinshausen, N. and BÜhlmann, P. (2010). Stability selection. J. R. Stat. Soc. Ser. B. Stat. Methodol. 72 417-473. MR2758523

[23] Neuvial, P. and RoQuain, E. (2012). On false discovery rate thresholding for classification under sparsity. Ann. Statist. 40 2572-2600. MR3097613

[24] Wainwright, M. J. (2009). Sharp thresholds for high-dimensional and noisy sparsity recovery using $\ell_{1}$-constrained quadratic programming (Lasso). IEEE Trans. Inform. Theory $\mathbf{5 5}$ 2183-2202. MR2729873

[25] Wasserman, L. and Roeder, K. (2009). High-dimensional variable selection. Ann. Statist. 37 2178-2201. MR2543689

[26] Zhang, A. Y. and ZHOU, H. H. (2016). Minimax rates of community detection in stochastic block models. Ann. Statist. 44 2252-2280. MR3546450

[27] ZhanG, C.-H. (2010). Nearly unbiased variable selection under minimax concave penalty. Ann. Statist. 38 894-942. MR2604701

[28] ZhaO, P. and Yu, B. (2006). On model selection consistency of Lasso. J. Mach. Learn. Res. 7 2541-2563. MR2274449

C. BUTUCEA

LAMA (UMR 8050), UPEM, UPEC, CNRS

UNIVERSITÉ PARIS-EST MARNE-LA-VALLÉE

F-77454, MARne-LA-VALLÉE

FRANCE

AND

CREST, ENSAE, UNIVERSITÉ PARIS-SACLAY

5, AVE. Henry le Chatelier

91120 Palaiseau CEDEX

FRANCE

E-MAIL: cristina.butucea@u-pem.fr

N. A. Stepanova

SCHOOL OF MATHEMATICS AND STATISTICS

CARLETON UNIVERSITY

OTTAWA, ONTARIO K1S 5B6

CANADA

E-MAIL: nstep@math.carleton.ca

\section{NDAOUD}

CREST, ENSAE, UNIVERSITÉ PARIS-SACLAY

5, AVE. HENRY LE CHATELIER

91120 Palaiseau CEDEX

FRANCE

E-MAIL: mohamed.ndaoud@ensae.fr

\author{
A. B. TSYBakov \\ CREST, ENSAE, UNIVERSITÉ PARIS-SACLAY \\ 5, AVE. HENRY LE CHATELIER \\ 91120 Palaiseau CedeX \\ FRANCE \\ E-MAIL: alexandre.tsybakov@ensae.fr
}

